\title{
SI IV Resonance Line Emission during Solar Flares: Non-LTE, Nonequilibrium, Radiation Transfer Simulations
}

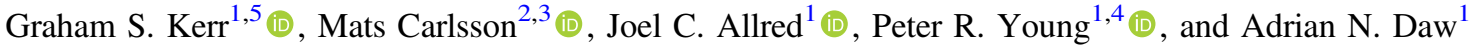 \\ ${ }^{1}$ NASA Goddard Space Flight Center, Heliophysics Sciences Division, Code 671, 8800 Greenbelt Rd., Greenbelt, MD 20771, USA; graham.s.kerr@ nasa.gov \\ ${ }_{2}^{2}$ Rosseland Centre for Solar Physics, University of Oslo, P.O. Box 1029, Blindern, N-0315 Oslo, Norway \\ ${ }^{3}$ Institute of Theoretical Astrophysics, University of Oslo, P.O. Box 1029, Blindern, N-0315 Oslo, Norway \\ ${ }^{4}$ College of Science, George Mason University, Fairfax, VA 22030, USA \\ Received 2018 October 3; revised 2018 November 20; accepted 2018 November 26; published 2019 January 18
}

\begin{abstract}
The Interface Region Imaging Spectrograph routinely observes the Si IV resonance lines. When analyzing quiescent observations of these lines, it has typically been assumed that they form under optically thin conditions. This is likely valid for the quiescent Sun, but this assumption has also been applied to the more extreme flaring scenario. We used 36 electron-beam-driven radiation hydrodynamic solar flare simulations, computed using the RADYN code, to probe the validity of this assumption. Using these simulated atmospheres, we solved the radiation transfer equations to obtain the non-LTE, nonequilibrium populations, line profiles, and opacities for a model silicon atom, including charge exchange processes. This was achieved using the "minority species" version of RADYN. The inclusion of charge exchange resulted in a substantial fraction of Si IV at cooler temperatures than those predicted by ionization equilibrium. All simulations with an injected energy flux $F>5 \times 10^{10} \mathrm{erg} \mathrm{cm}^{-2} \mathrm{~s}^{-1}$ resulted in optical depth effects on the Si IV emission, with differences in both intensity and line shape compared to the optically thin calculation. Weaker flares (down to $F \approx 5 \times 10^{9} \mathrm{erg} \mathrm{cm}^{-2} \mathrm{~s}^{-1}$ ) also resulted in Si IV emission forming under optically thick conditions, depending on the other beam parameters. When opacity was significant, the atmospheres generally had column masses in excess of $5 \times 10^{-6} \mathrm{~g} \mathrm{~cm}^{-2}$ over the temperature range 40-100 kK, and the Si IV formation temperatures were between 30 and $60 \mathrm{kK}$. We urge caution when analyzing Si IV flare observations, or when computing synthetic emission without performing a full radiation transfer calculation.
\end{abstract}

Key words: line: formation - methods: numerical - radiative transfer - Sun: flares - Sun: transition region - Sun: UV radiation

\section{Introduction}

The solar transition region (TR) is the thin layer through which the atmosphere steeply transitions from chromospheric temperatures, densities, and ionization fractions to coronal values. This means that the temperature climbs through several $\times 10-100 \mathrm{kK}$ to the $>1$ MK corona, densities precipitously fall from $\approx 10^{11}$ to $\approx 10^{8-9} \mathrm{~cm}^{-3}$, and the atmosphere changes from a partially ionized state to being almost entirely ionized. The TR is optically thin to most radiation, with notable exceptions being the $\mathrm{C}$ II resonance lines that form at the base of the TR (Rathore \& Carlsson 2015).

Solar flares release tremendous amounts of magnetic energy (up to $\approx 10^{32-33} \mathrm{erg}$ in the span of several minutes), which is subsequently transported from the release site in the corona to the lower atmosphere. It is believed that flare energy is transported by large numbers of electrons that are accelerated out of the thermal background (Brown 1971; Hoyng et al. 1976). These nonthermal electrons are ducted along magnetic field lines, precipitating through the TR into the chromosphere, where they lose their energy via Coulomb collisions (Brown 1971; Emslie 1978; Holman et al. 2011). Flares are characterized by intense, broadband enhancements to the solar radiative output, the bulk of which originates from the chromosphere (Fletcher et al. 2011), due to plasma heating and ionization. Heating drives mass motions with upflows ("chromospheric evaporation"), filling the flux tubes with

\footnotetext{
${ }^{5}$ NPP Fellow, administered by USRA.
}

denser chromospheric material, and downflows ("chromospheric condensations"), the properties of which are sensitive to the location and magnitude of flare heating (e.g., Doschek et al. 1980; Fisher et al. 1985a, 1985b, 1985c; Fisher 1989; Milligan et al. 2006; Milligan \& Dennis 2009; Graham \& Cauzzi 2015). It is worth noting that additional energy transport mechanisms have recently been proposed, for example, the dissipation of high-frequency Alfvénic waves (Fletcher \& Hudson 2008; Kerr et al. 2016; Reep \& Russell 2016; Reep et al. 2018b).

The extreme conditions through the TR make it an important interface for energy, mass, and radiation transport. The sensitivity of the numerous spectral lines produced by ions in the TR to local plasma conditions makes the TR a source of rich diagnostic potential of flare energy transport. For example, the Doppler motions of spectral lines resulting from chromospheric evaporations or condensations are a useful, and commonly used, observation with which to make a modeldata comparison to critically attack models of flare energy transport. It is crucial to accurately forward-model the radiation that is produced by a flare simulation.

Two such lines that are now routinely observed by the Interface Region Imaging Spectrograph (IRIS; De Pontieu et al. 2014) are the Si IV resonance lines at 1393.75 and $1402.77 \AA$. These lines have been exploited to study various solar phenomenon since the launch of IRIS, including flares, and are often analyzed using the simplifying assumption that they are formed under optically thin conditions. This is likely a safe assumption for many situations. However, during 
impulsive heating events, such as solar flares, temperatures and densities can increase substantially over the quiet-Sun values, potentially increasing the atmospheric opacity at these wavelengths. If the lines form under conditions where opacity effects are non-negligible, then interpreting observations, particularly Doppler motions or other velocity effects, become more complex (e.g., Kuridze et al. 2015; Kerr et al. 2016; Brown et al. 2018).

Flare observations of the Si IV resonance lines have largely focused on investigating atmospheric flows, and while these lines have been analyzed under the optically thin assumption, there have been (albeit ambiguous) hints that opacity effects may be present.

Tian et al. (2015) noted that the lines broadened and exhibited redshifts in flare ribbon sources of up to a few $\times 10$ to $>100 \mathrm{~km} \mathrm{~s}^{-1}$ (which is surprisingly fast), predominately appearing as asymmetries in the red wing, and not as a shift of the whole line. Other studies noted instead that the lines were fully redshifted to a few $\times 10 \mathrm{~km} \mathrm{~s}^{-1}$ (Warren et al. 2016b; Li et al. 2017). Li et al. (2017) pointed out that the Si IV can deviate strongly from a Gaussian shape in the flare ribbons, with some profiles suitable for fitting with a Gaussian function but others not. In a very small flare (B4 class) Warren et al. (2016b) found that the Si IV intensity increased by up to $1000 \times$, with a line width similar in magnitude to the optically thick C II lines. They also noted that the Si IV emission showed persistent redshifts, in contrast to the Mg II redshifts, which were bursty in character.

Brannon et al. (2015) found oscillations in Si IV observations from flare ribbons. They noted that while line ratios suggested an optically thin plasma (in the optically thin limit the intensity ratio of the $\lambda 1393.75$ to $\lambda 1402.77$ line should be equal to 2 , the ratio of their oscillator strengths), some pixels deviated from the optically thin value. Further, while not interpreted as such, some of the profiles shown in their Figure 7 could show signs of self-absorption features.

High-Resolution Telescope Spectrometer (Bartoe \& Brueckner 1975) observations of a light bridge spectrum from 1978 February 13 showed an Si IV resonance line ratio of 10:1, far from the optically thin limit (Brekke et al. 1991). It was noted by Jaeggli et al. (2018) that an X1 flare was produced from the same region, that this flare had an unusually long decay phase, and that there were several smaller events in the region. They speculate that the light bridge spectrum was actually the result of ongoing flaring activity and the anomalous intensity ratio was due to flares.

To our knowledge no other solar flare measurements of the $\lambda 1393.75 / \lambda 1402.77$ ratio have been reported (for telemetry reasons not every observation from IRIS includes both resonance lines in the linelist). There have been some stellar flare observations of the Si IV resonance line ratio (and other TR line ratios) that indicate the presence of opacity effects (Mathioudakis et al. 1999; Bloomfield et al. 2002; Christian et al. 2006). Mathioudakis et al. (1999) relate this to an electron density enhancement during the flare up to $\approx 10^{11.5} \mathrm{~cm}^{-3}$, using an escape probabilities argument. If stellar flare observations indicate optical depth effects, then it is certainly prudent to consider this in the solar case, though the atmospheric structure of $\mathrm{M}$ dwarf stars such as $\mathrm{YZ} \mathrm{CMi,}$ studied by Mathioudakis et al. (1999), is rather different from the solar atmosphere.
Opacity effects on the Si IV lines have been suggested in regard to other solar phenomena, such as UV bursts. These are smaller-scale impulsive heating events, thought to be caused by reconnection in the TR or chromosphere. There has recently been a healthy debate as to both their origin and their relation to Ellerman bombs (e.g., Peter et al. 2014; Judge 2015; Young et al. 2018b). Self-absorption effects during a UV burst were reported by Yan et al. (2015), alongside a resonance line intensity ratio of 1.7. Bi-directional flows were ruled out as the origin of the central reversal feature. Similar observations were reported by Nelson et al. (2017).

As well as the ratio of the resonance lines, the ratio of the Si IV lines to O IV lines (also observed by IRIS) offers a density diagnostic, though, as discussed by Judge (2015), Doschek et al. (2016), and Young et al. (2018a), it is important to understand certain caveats with regard to nonequilibrium ionization and dependence on formation temperatures. We note this diagnostic here as it has become a fairly commonly used tool, but if opacity effects are non-negligible, the utility of this ratio is ambiguous.

It is common in both flare, burst, and more quiescent modeling to assume that the Si IV emission is optically thin, so that atomic databases such as CHIANTI (Dere et al. 1997; Landi et al. 2013) are used, along with a modeled atmospheric structure or differential emission measure model, to compute the emissivity without performing full radiation transfer. Examples include Olluri et al. (2015), Martínez-Sykora et al. (2016), and Nóbrega-Siverio et al. (2017), who used radiation magnetohydrodynamic modeling to study various solar features. They found reasonable agreement with IRIS observations, particularly when nonequilibrium ionization effects were included.

The effects of photoexcitation, nonequilibrium ionization, kappa-distributions (rather than Maxwellian), and high-energy tails of electron distributions on the formation of Si IV and O IV have been investigated in detail by Dudík et al. (2014, 2017), Dzifčáková et al. (2016, 2017), and Dzifčáková \& Dudík (2018). They noted that to be able to reproduce observed ratios of Si IV $\lambda 1402.77$ to O IV $\lambda 1401.2$, inclusion of nonequilibrium effects was crucial. Low-temperature shoulders were present in the ionization fractions of Si IV ions compared to the assumption of ionization equilibrium.

In flares the $\mathrm{Si}$ IV emission has been computed in a similar manner to investigate the flows resulting from multithreadedloop simulations versus single-loop simulations (Reep et al. 2016, 2018a), where it was found that multithreaded loops with heating durations on the order of $50-100 \mathrm{~s}$ are required to reproduce the observed persistent redshifts. Also, Polito et al. (2018) synthesized Si IV from electron-beam-driven nanoflare simulations or from simulations with in situ energy deposition, noting that observed Doppler motions were only consistent with the electron beam scenario (and then only for certain nonthermal electron distribution parameters).

We could find only one flare model in which the Si IV emission was computed in an alternative way, where optical depths were included in a simplified manner (Dame \& Vial 1985). Using a semi-empirical flare atmosphere, Dame \& Vial (1985) modeled the Si IV lines, finding them to be formed in optically thick conditions, significantly more intense than the typical quiet-Sun profiles, and with a deep central reversal feature. 
Since the formation properties of the Si IV resonance lines in flare models that take account of potential opacity effects have been explored relatively little in comparison to other IRIS lines, such as the Mg II $h$ and $k$ lines (e.g., Kerr et al. 2016; Kerr 2017; Rubio da Costa \& Kleint 2017), we present the initial results from a survey of electron-beam-driven solar flares in which the Si IV resonance lines were computed in detail. This is a prudent investigation given hints of opacity effects reported in flares or other heating events, and given the importance of the Si IV lines as a means to understand energy transport and mass motions. Additionally, it is important to understand whether our flare models are able to reproduce not just one observation but many, so combining Si IV with the other spectral lines observed by IRIS can assess the validity of flare models.

The numerical approach, model atom, and flare simulations are introduced in Section 2. Si IV resonance line emission is discussed in Section 3, where the line profiles, light curves, and ratios are presented, along with details regarding opacity and the atmospheric properties in the line formation region. In Section 3.3 the Si IV lines are placed in context with other IRIS observables (Mg II $k$ line, Mg II $\lambda 2791$ line, C II $\lambda 1334$ line, and O I $\lambda 1356$ line). We summarize our findings in Section 4.

\section{Numerical Simulations}

\subsection{The RADYN Code}

The radiation hydrodynamics code RADYN (Carlsson \& Stein 1992, 1997) is a well-established resource with which to study the dynamics of the flaring atmosphere. It has the facility to model flares via an injection of nonthermal particles (Abbett \& Hawley 1999; Allred et al. 2005, 2015), and more recently via dissipation of Alfvénic waves (Kerr et al. 2016). We present a brief introduction here, but see Allred et al. (2015) for a more detailed description. RADYN solves the coupled, nonlinear equations of hydrodynamics, radiation transport, and timedependent (nonequilibrium) atomic level populations in a 1D plane-parallel atmosphere, on an adaptive grid (Dorfi \& Drury 1987), that represents one leg of a symmetric flux tube.

Typically, the detailed non-LTE (NLTE), radiation transport is solved for three elements that are considered important for the energy balance in the chromosphere $(\mathrm{H}, \mathrm{He}$, and $\mathrm{Ca})$, with continua from other species treated in LTE as background metal opacities using the Uppsala opacity package (Gustafsson 1973). Optically thin losses are included by summing all transitions from the CHIANTI atomic database, apart from those transitions solved in detail. Additional back-warming and photoionizations by soft X-ray, extreme-ultraviolet, and ultraviolet radiation are included as described in Allred et al. (2015).

Radiation transport is computed assuming complete redistribution (CRD), which may overestimate radiation losses from certain transitions (Uitenbroek 2002). We mitigate the effects of not employing the more physically realistic (but computationally expensive) partial redistribution (PRD) by truncating the Lyman lines at 10 Doppler widths and by omitting the $\mathrm{Mg}$ II $h$ and $k$ lines (which in part counters the overestimation of losses from the $\mathrm{Ca}$ II $\mathrm{H}$ and $\mathrm{K}$ lines). Thermal conduction is a modified form of Spitzer conductivity such that it saturates to avoid exceeding the electron free-streaming limit (Smith \& Auer 1980).
When injecting flare energy via nonthermal electron beams, the electron distribution is computed by solving the FokkerPlanck equations (note that we do not include return current effects in these simulations). The nonthermal electron distribution is described by a power-law energy spectrum with total energy flux $F$ erg $\mathrm{cm}^{-2} \mathrm{~s}^{-1}$ above a low-energy cutoff $E_{c} \mathrm{keV}$, with spectral index $\delta$. Direct nonthermal collisional excitations and ionizations from the ground state of hydrogen and helium by the electron beams are included. We use the expressions from Fang et al. (1993).

The solution from RADYN (where $\mathrm{H}, \mathrm{He}$, and $\mathrm{Ca}$ are solved in detail, and heating and cooling by their radiation affect the thermodynamic response) can then be used to solve the timedependent, NLTE, radiation transport and nonequilibrium atomic level populations for a "minority," or "trace," species not considered in detail during the initial simulation. This approach uses the hydrodynamic variables at each time step, stored from the initial full solution, performing only the radiation transport calculations with the assumption that the thermodynamics are unaffected by the resulting radiation. The minority species is removed from the background opacity package so as to avoid double counting. Such an approach was used by Judge et al. (2003) to investigate basal emission using C II. We employ this "minority species" version of RADYN here to investigate Si IV emission during solar flares, and we refer to this as MS_RADYN hereafter so as to avoid confusion with the full RHD RADYN flare simulations. A point to note here is that we save the hydrodynamic variables required for use in MS_RADYN at every time step used internally during the original RHD solution, so the Si IV computation is not constrained by the time interval selected by the user for the main RADYN output.

\subsection{Model Silicon Atom}

A 30-level-with-continuum silicon atom that included the ground terms of Si I, Si II, Si III, Si IV, and Si V, along with several excited levels of each charge state, was used to model 31 bound-bound transitions and 33 bound-free transitions. The major differences between our model atom and the CHIANTI approach are the inclusion of nonequilibrium ionization, photoionizations plus detailed radiative recombinations, charge exchange processes, and the potential effects of opacity. Details of the construction of this model atom are presented in Appendix A.

Comparing the ionization fractions at $t=0 \mathrm{~s}$ in an MS_RADYN simulation to those predicted by the ionization equilibrium in CHIANTI reveals that the inclusion of photoionizations plus detailed radiative recombinations, as well as charge exchange in our model atom, results in an increased fraction of Si IV at lower temperatures, a higher and broader peak, and a shift to a somewhat lower peak temperature of $\log T=4.84(T=69.6 \mathrm{kK})$ compared to the CHIANTI equilibrium ionization value of $\log T=4.9$ $(T=79.4 \mathrm{kK})$. Other differences are present in Si I, SI II, and Si III also. This is shown in Figure 1(a), where the dashed lines are the CHIANTI ionization equilibrium fractions and the solid lines are the MS_RADYN fractions. Removing charge exchange yields ionization fractions that are very similar to CHIANTI (Figure 1(b)), highlighting the importance of this process. If we do not include charge exchange or photoionizations, treating recombinations and ionizations as in CHIANTI, then we obtain ionization fractions that match 

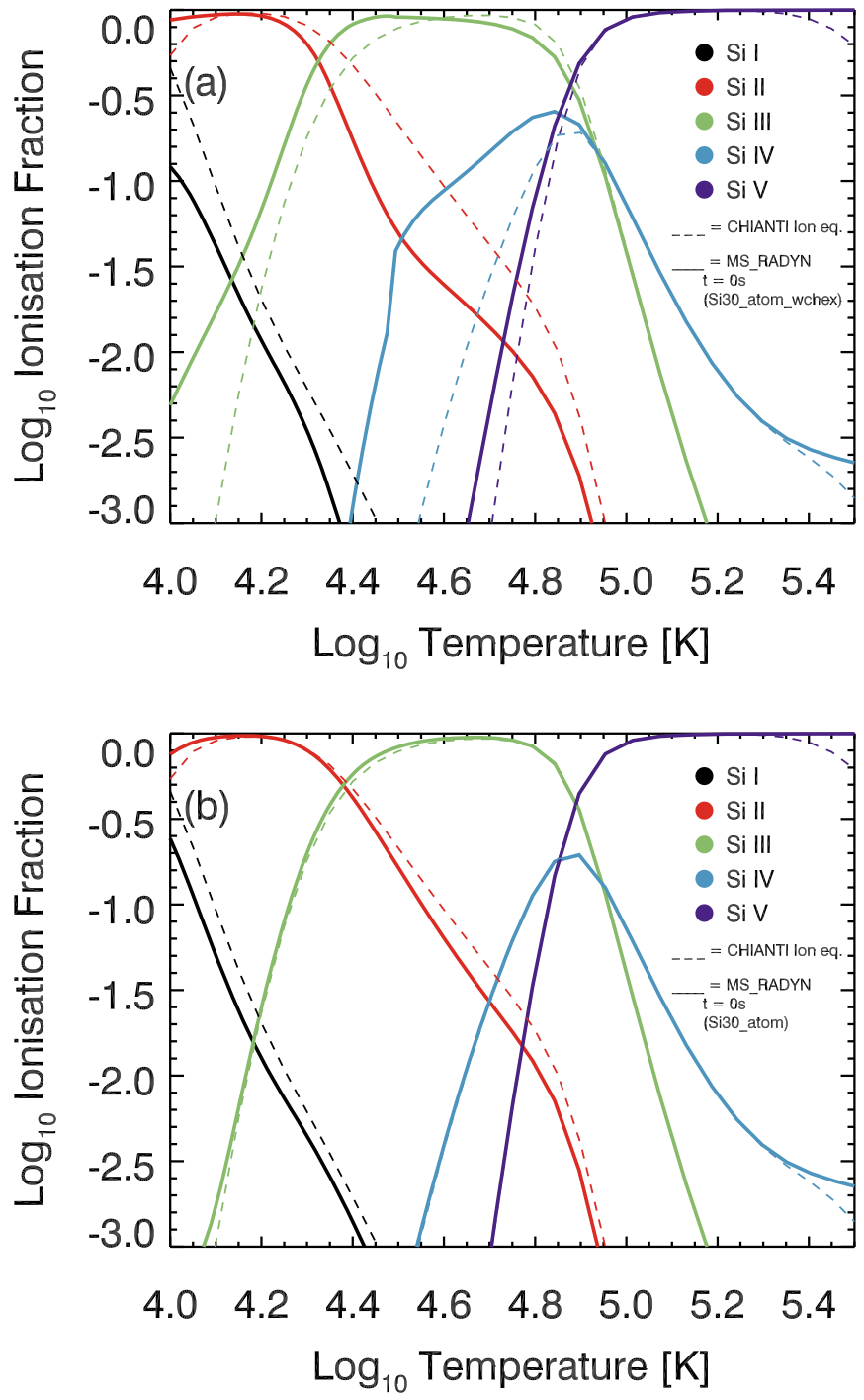

Figure 1. $\mathrm{Si}$ ionization fractions as functions of temperature from an MS_RADYN calculation (solid lines) and from CHIANTI (dashed lines). Si I (black), Si II (red), Si III (green), Si IV (blue), and Si V (purple) are shown. Panel (a) shows the case where charge exchange is included, where Si IV peaks at $\log T=4.84$ in MS_RADYN, with more emission from cooler layers, and at $\log T=4.90$ from CHIANTI. Panel (b) is the case without charge exchange, illustrating the importance of this process.

CHIANTI. Hereafter we refer to the 30-level-with-continuum model atom that includes charge exchange as Si30_atom wchex and the model atom that excludes charge exchange (but which is otherwise the same) as Si30_atom. Unless specified, all results use Si30_atom_wchex, though we at times show Si30_atom results for illustration of the differences. Since charge exchange is a process dependent on the density of hydrogen or helium and their ions, the ionization fractions will vary somewhat with different preflare atmospheres.

\subsection{Parameter Survey}

Thirty-six flare simulations were produced, covering a wide range of energy fluxes and several typical values of $E_{c}$ and $\delta$, in order to assess the effect of both the strength of energy injection and the location of peak deposition on the formation of the SiIV resonance lines. Flare energy was injected into a $10 \mathrm{Mm}$ loop spanning the subphotosphere through to the corona, which was initially in radiative equilibrium. The temperature at the loop apex was $T=$ $3.2 \mathrm{MK}$, and the hydrogen number density was $n_{\mathrm{H}}=6.6 \times$ $10^{9} \mathrm{~cm}^{-3}$. The photospheric temperature was $T=5800 \mathrm{~K}$, and the TR was initially located at an altitude of $z \approx 1.156 \mathrm{Mm}$ (column mass, $c_{\text {mass }} \approx 2.25 \times 10^{-5} \mathrm{~g} \mathrm{~cm}^{-2}$ ). To maintain coronal and photospheric energy balance, additional nonradiative heating was applied to grid cells with column mass $c_{\text {mass }}<1 \times 10^{-6} \mathrm{~g} \mathrm{~cm}^{-2}$ and $c_{\text {mass }}>7.6 \mathrm{~g} \mathrm{~cm}^{-2}$. To mimic the effect of incoming disturbances from the other leg of the flaring loop, we employ a reflecting boundary condition at the loop apex, while a transmitting boundary was used at the base. The pre-flare atmosphere is shown as the gray shaded portions of Figure 2.

Using the notation $X F Y$ to mean $X \times 10^{Y} \mathrm{erg} \mathrm{cm}^{-2} \mathrm{~s}^{-1}$, the electron beam parameters simulated were $F=[5 \mathrm{~F} 8,1 \mathrm{~F} 9,5 \mathrm{~F} 9$, $1 \mathrm{~F} 10,5 \mathrm{~F} 10,1 \mathrm{~F} 11], \delta=[4,6]$, and $E_{c}=[20,30,40] \mathrm{keV}$. Energy was injected at a constant rate for $t=10 \mathrm{~s}$, and the atmospheres were allowed to cool for a subsequent $40 \mathrm{~s}$. A moderate to strong flare might have an energy flux on the order of $>5 \mathrm{~F} 10$, weaker flares on the order of $\approx 1 \mathrm{~F} 9-1 \mathrm{~F} 10$, and microflares (or smaller heating events) on the order of $<1 \mathrm{~F} 9$. Our simulations therefore cover a wide range of heating events (of course, if the typical flare footpoint sizes have been underestimated owing to the spatial resolution of observations, then our stronger flare may actually represent only modest flares). Hereafter we refer to simulations as, for example, $1 \mathrm{~F} 11 \delta 4 E_{c} 20$ to mean a simulation with parameters $F=1 \times 10^{11} \mathrm{erg} \mathrm{cm}^{-2} \mathrm{~s}^{-1}, E_{c}=$ $20 \mathrm{keV}$, and $\delta=4$.

Each of the flare simulations was then used as input along with the Si atom Si30_atom_wchex described in Section 2.2 in the MS_RADYN code.

\subsection{Atmospheric Response}

In each simulation the injection of flare energy resulted in temperature and electron density enhancements. This resulted in an increased population of Si IV, which occurred more rapidly in the stronger flares. Figure 2 summarizes the atmospheric response, showing the temperature, electron density, Si IV ionization fraction, and the population of the Si IV $\lambda 1402.77$ upper level, as functions of column mass at $t=5 \mathrm{~s}$.

The weaker flares produced only modest enhancements where chromospheric temperatures barely exceed $T \approx 6-10 \mathrm{kK}$ and electron densities reached a few $\times 10^{12} \mathrm{~cm}^{-3}$ in the upper chromosphere. In these simulations the temperature enhancement is not sufficient to produce a substantial increase of the $\mathrm{Si} I V$ fraction. It is clear that the stronger flares produced a much more dramatic response, with temperatures exceeding $T=20 \mathrm{kK}$ to $T>50 \mathrm{kK}$ in the mid- to upper flare chromosphere. Note that here we are defining the flare chromospheres as the regions below which the temperatures climb, and electron densities drop precipitously through the TR to the corona (i.e., below $T \gtrsim 100 \mathrm{kK}$ and $n_{e} \lesssim 10^{10} \mathrm{~cm}^{-3}$ ). The enhanced temperature over a wider swathe of the chromosphere and TR results in a substantial increase of the Si IV populations, such that the formation location of the Si IV resonance lines shifts lower in altitude.

The thickness of the atmosphere over which Si IV populations are enhanced changes over time. Simulations that initially have a high Si IV fraction over a large geometric region may 

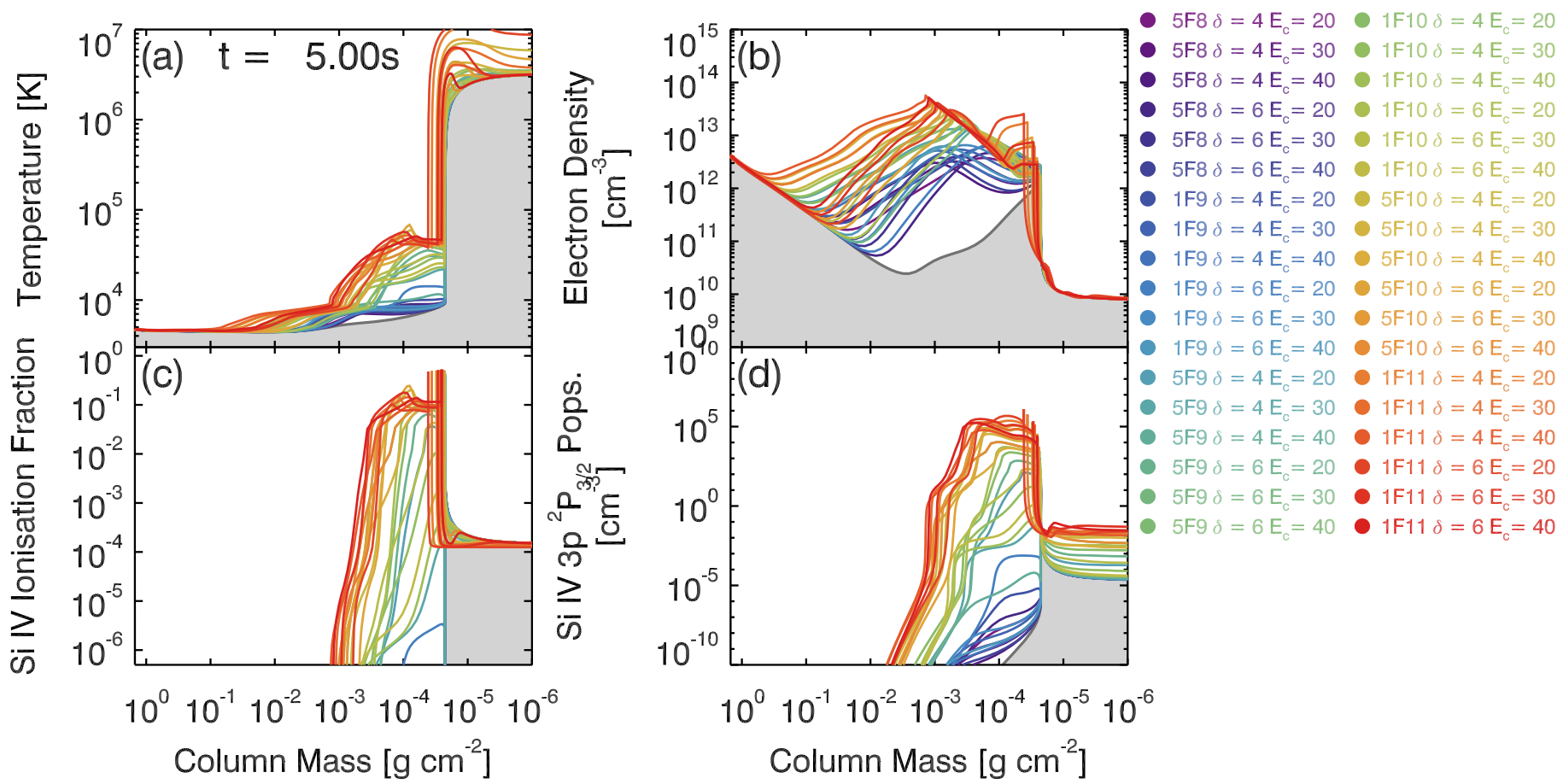

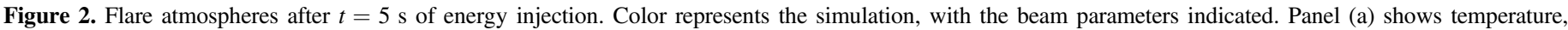

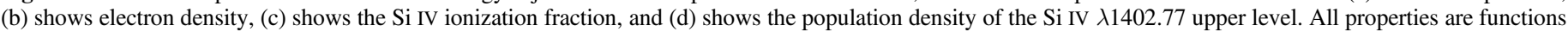

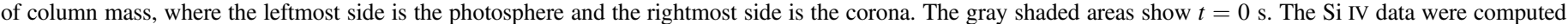
using MS_RADYN with Si30_atom_wchex.

end with only a narrow layer of enhanced Si IV if the atmosphere compresses. The opposite can also be true, so that some simulations build more slowly to a sufficient temperature and density structure to allow the fraction of Si IV to increase. Generally speaking, the larger the energy injected is, and the softer the electron beam is (that is, the higher proportion of lower-energy electrons that deposit their energy in the upper atmosphere), then the larger the enhancement to the Si IV density.

\section{Formation of Si IV in Flares}

\subsection{Line Profiles and Ratios}

To compare the results of solving the full NLTE, nonequilibrium radiation transfer problem, rather than producing synthetic line profiles assuming a priori that they are optically thin, we show the resonance line profiles, ratios, and light curves computed via each method.

To compute the optically thin case, which we refer to as Model $\mathrm{B}$ hereafter, we used the following procedure. The contribution function, $G\left(\lambda, n_{e}, T\right)\left(\operatorname{erg~cm}^{-3} \mathrm{~s}^{-1} \mathrm{sr}^{-1}\right)$, allows the calculation of the intensity emitted at each depth point $z$ in our flare atmospheres, $I_{\lambda z}$. These were constructed using standard CHIANTI software and contain information about various atomic properties (ionization and recombination rates, etc.). Ionization equilibrium was assumed here. $G\left(\lambda, n_{e}, T\right)$ values were obtained from the atomic data in CHIANTI V8.0.7 and were tabulated with a resolution of $\delta \log T=0.05$ and $\delta \log n_{e}=0.5$. At each grid cell and each time in the RADYN atmospheres we interpolate $G\left(\lambda, n_{e}, T\right)$ to the electron density and temperature in that grid cell, and then we compute $I_{\lambda z}$ by

$$
I_{\lambda z}=A_{b} G\left(\lambda, n_{e}, T\right) n_{e}(z) n_{\mathrm{H}}(z) \delta z,
$$

where $A_{b}$ is the abundance (we used photospheric abundance from Asplund et al. 2009; see Appendix B), $\delta z$ is the size of the grid cell, and $n_{\mathrm{H}}$ is the hydrogen density. Within each grid cell the line is thermally broadened using the local temperature and Doppler-shifted by any velocity gradients. The intensity is then the sum of the contribution from each grid cell along the whole loop, $I_{\lambda}=\sum_{z} I_{\lambda z}$. In the wavelength range selected, only the $G\left(\lambda, n_{e}, T\right)$ values associated with the resonance line transitions were considered. Other species/transitions that contribute to the solar spectrum within that wavelength range were omitted. This will not affect the intensity of Si IV produced but permits a simpler analysis of line ratios and comparison to MS_RADYN results, which also do not include blends from other species.

For both methods we show results from disk center. We assume no geometric effects on Doppler shifts since Si IV forms relatively low in the atmosphere in relation to the length of the loop, and in a narrow region (i.e., we assume that the portion of the loop in which the resonance lines originate is vertical).

Resonance lines profiles at $t=0 \mathrm{~s}$ from each method are shown in Figure 3. Panel (a) shows the case with charge exchange, whereas panel (b) shows the case without. While there are intensity differences between the two models when charge exchange is included, the line shapes are comparable. In both cases the resonance line ratio $R_{\text {res }}=I_{1393} / I_{1402} \approx 2$, as would be expected in the optically thin scenario. Excluding charge exchange results in a very close match between MS_RADYN and Model B.

During the flares, the Si IV resonance lines exhibit changes in line intensity, shape, and Doppler motions. These changes 

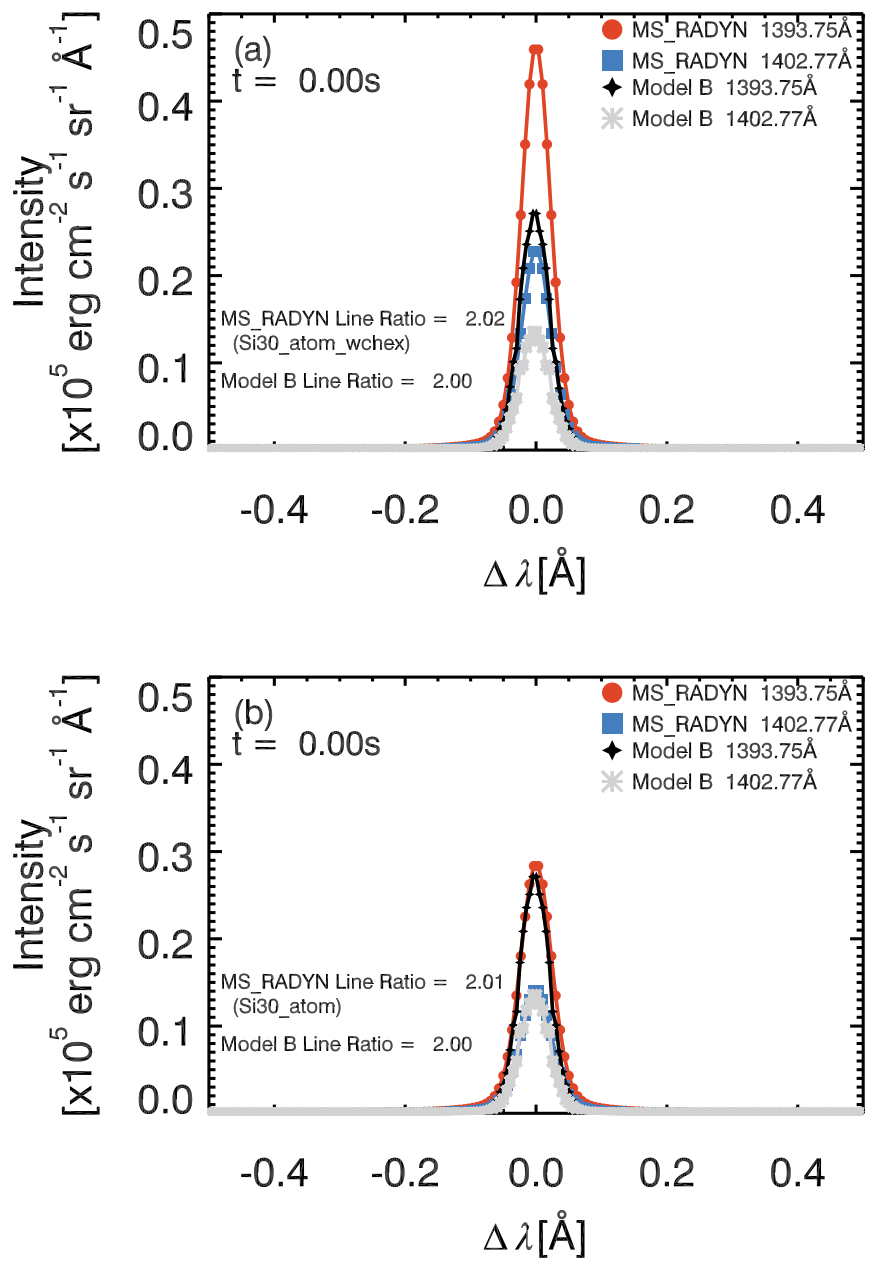

Figure 3. Comparing resonance line profiles from MS_RADYN (red circles: $1393 \AA$ A blue squares: $1402 \AA$ ) and CHIANTI (black diamonds: $1393 \AA$ A; gray stars: $1402 \AA$ ) at $t=0 \mathrm{~s}$ in our simulations. Panel (a) shows MS_RADYN results using model atom Si30_atom_wchex that includes charge exchange. Panel (b) shows model atom Si30_atom that excludes charge exchange.

range from modest in the lower-energy simulations to dramatic in the higher-energy flares. Figure 4 illustrates the effect of varying the injected energy flux for simulations with fixed spectral index and low-energy cutoff $\left(\delta=4\right.$ and $\left.E_{c}=20 \mathrm{keV}\right)$ on the $\lambda 1402.77$ line. This figure also shows the differences that result from using either Model B or MS_RADYN to synthesize the line. For each of the six values of energy flux two pairs of images are presented showing a stackplot of line intensity as functions of wavelength and time. The left-hand image of each pair shows the line computed via Model B, and the right-hand image shows the line computed by MS_RADYN. The color scale varies from panel to panel so as to illustrate the evolution of each simulation over the large intensity range present between the weaker and stronger flares. The sharp discontinuity in some panels at $t \sim 10 \mathrm{~s}$ is due to the rapid cooling of the atmosphere following cessation of the electron beam. In those cases the flare enhancements were so strong that the scaling on the figures makes it difficult to see the cooling phase.

For 5F8 the response is very similar between the two techniques, with intensity enhancements, slight blueshifts, but overall a fairly symmetric line profile. The MS_RADYN profile is somewhat more intense. Similarly, the 1 F9 simulation produces fairly comparable profiles, albeit with an increased intensity difference. As discussed later, in these simulations the MS_RADYN computed emission is optically thin, with differences resulting from using a full radiation transfer approach.

Further increasing the energy flux does eventually lead to marked differences between the two techniques. In the 5F9, 1F10, 5F10, and 1F11 simulations Model B produced Si IV emission that is much weaker than the equivalent MS_RADYN profiles (by an order of magnitude in some cases), is more symmetric, and is, generally, single peaked. In contrast, the MS_RADYN Si IV emission is broader, with multiple line components, self-absorption features, and strong asymmetries, and does not always share the same character of Doppler shift as the Model B profiles. The profiles are generally flatter, with wider cores. These difference arise because the Si IV emission is affected by optical depth effects in these more energetic simulations. For these electron beam parameters, there is a transition from blueshift to redshift that occurs for injected fluxes $>1 \mathrm{~F} 10$.

The effects of varying the other nonthermal beam parameters are presented in Figure 5, focusing only on the MS_RADYN results. The top row shows $\delta=4$ and the bottom row $\delta=6$, with $E_{c}=20,30,40 \mathrm{keV}$ from left to right, and with the injected energy fixed at $1 \mathrm{~F} 10$. Here we show the Si IV $\lambda 1393.75$ line. At this value of injected energy flux the atmosphere at the heights at which Si IV forms is upflowing, so that all of the emission here is blueshifted to some degree. For $\delta=4$ and $E_{c}=20 \mathrm{keV}$ the emission shows self-absorption and asymmetries. As the low-energy cutoff increases (i.e., energy is carried by more energetic, deeply penetrating electrons), the main atmospheric response occurs somewhat deeper in the atmosphere. The lines become narrower, weaker, and single peaked. Similarly, the bottom row of Figure 5 shows that the beam parameters that would heat the upper atmosphere $\left(\delta=6\right.$ with $\left.E_{c}=[20,30]\right)$ exhibit high-intensity, selfabsorbed Si IV emission with asymmetries. Those profiles are wider than the $\delta=6$ with $E_{c}=40 \mathrm{keV}$ case. As we discuss in Section 3.2, the opacity effects are dependent on the location of heating. For some profiles shown in Figure 5, such as $\delta=6$ with $E_{c}=30 \mathrm{keV}$, it takes a few seconds for the signatures that are indicative of optical depth effects to appear.

To compare all of the simulations, we show light curves of integrated line intensity, as well as line intensity ratios, in Figure 6 (which shows the MS_RADYN results) and Figure 7 (which shows the Model B results), for all 36 simulations. Panel (a) in each figure shows the $1393.75 \pm 0.5 \AA$ integrated intensities on a log scale to show all of the simulations. Panel (b) in each figure shows the ratio $R_{\text {res }}=I_{1393} / I_{1402}$. As before, color represents the individual simulations.

It is clear that in both models the intensities scale considerably in magnitude with the level of injected flare energy flux. Generally the temporal behavior is shared among both the MS_RADYN and Model B derived profiles, where profiles typically peak at the end of the heating phase and rapidly decline after the cessation of the electron beams. For those simulations in which the Si IV light curves peak earlier, or which show more structure, there is some disagreement between Model $B$ and MS_RADYN. For example, there are peaks near $t \approx 5.5 \mathrm{~s}$ in Figure 6 and at $t \approx 2 \mathrm{~s}$ in Figure 7 . 


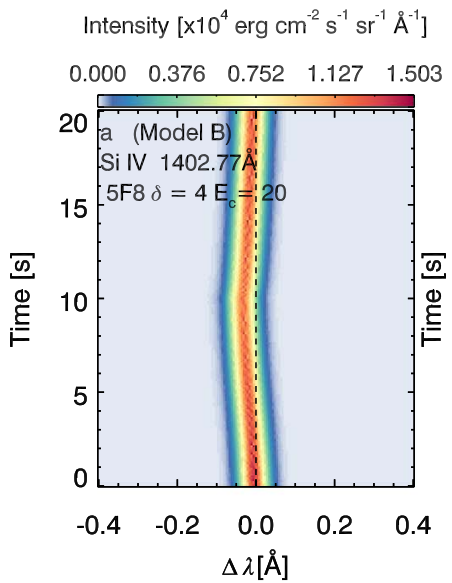

Intensity $\left[\times 10^{5} \mathrm{erg} \mathrm{cm}^{-2} \mathrm{~s}^{-1} \mathrm{sr}^{-1} \AA^{-1}\right]$

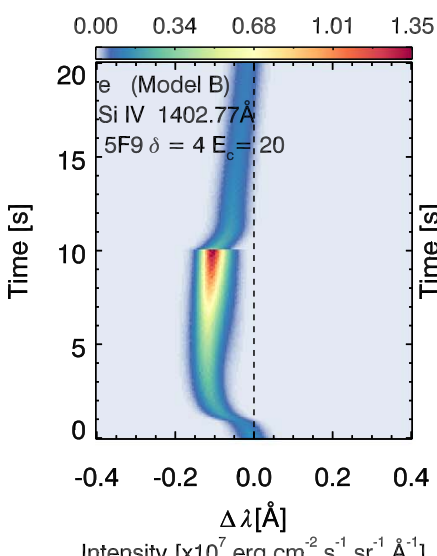

Intensity $\left[\times 10^{7} \mathrm{erg} \mathrm{cm}^{-2} \mathrm{~s}^{-1} \mathrm{sr}^{-1} \AA^{-1}\right]$

$\begin{array}{lllll}0.00 & 0.86 & 1.73 & 2.59 & 3.46\end{array}$

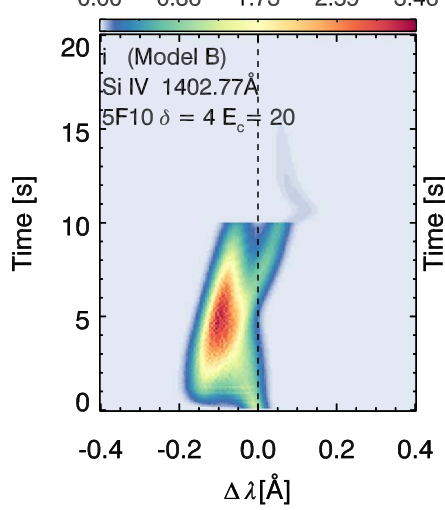

Intensity $\left[\times 10^{4} \mathrm{erg} \mathrm{cm}^{-2} \mathrm{~s}^{-1} \mathrm{sr}^{-1} \AA^{-1}\right]$

$\begin{array}{lllll}0.00 & 0.73 & 1.46 & 2.19 & 2.93\end{array}$

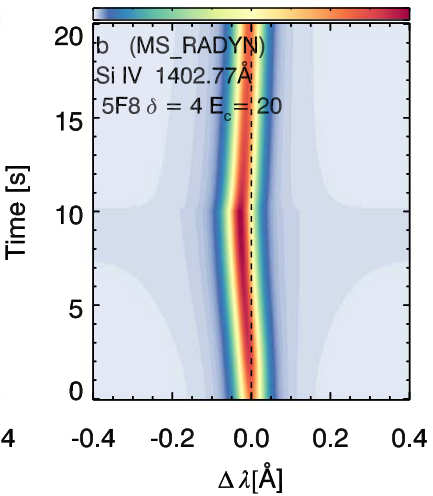

Intensity $\left[\times 10^{7} \mathrm{erg} \mathrm{cm}^{-2} \mathrm{~s}^{-1} \mathrm{sr}^{-1} \AA^{-1}\right]$

$\begin{array}{lllll}0.00 & 0.26 & 0.52 & 0.78 & 1.04\end{array}$

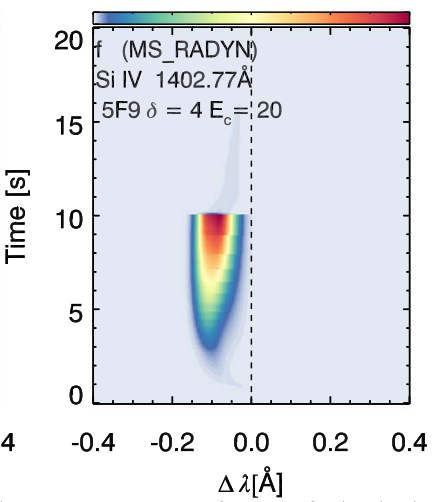

Intensity $\left[\times 10^{8} \mathrm{erg} \mathrm{cm}^{-2} \mathrm{~s}^{-1} \mathrm{sr}^{-1} \AA^{-1}\right]$

$\begin{array}{lllll}0.00 & 0.76 & 1.52 & 2.28 & 3.04\end{array}$

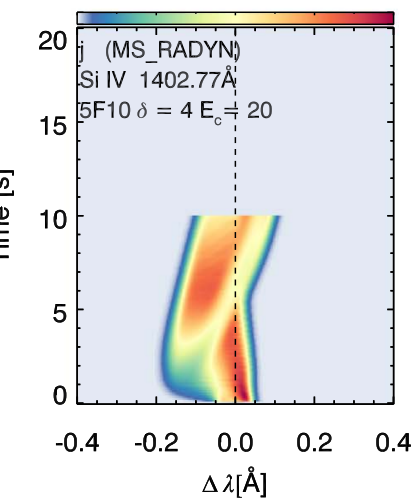

Intensity $\left[\times 10^{4} \mathrm{erg} \mathrm{cm}^{-2} \mathrm{~s}^{-1} \mathrm{sr}^{-1} \AA^{-1}\right]$

Intensity $\left[\times 10^{4} \mathrm{erg} \mathrm{cm}^{-2} \mathrm{~s}^{-1} \mathrm{sr}^{-1} \AA^{-1}\right]$

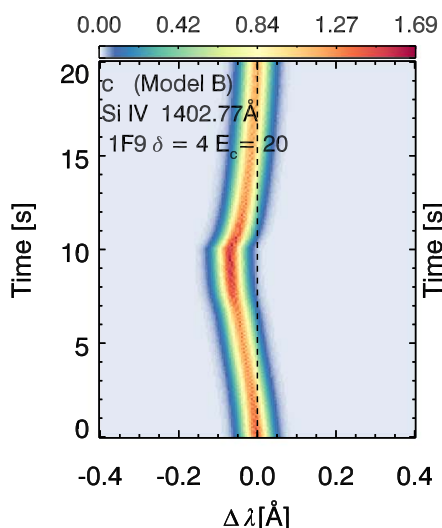

Intensity $\left[\times 10^{6} \mathrm{erg} \mathrm{cm}^{-2} \mathrm{~s}^{-1} \mathrm{sr}^{-1} \AA^{-1}\right]$

$\begin{array}{lllll}0.00 & 1.15 & 2.30 & 3.46 & 4.61\end{array}$

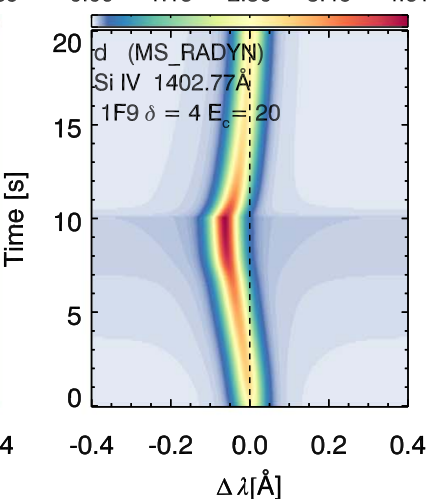

Intensity $\left[\times 10^{7} \mathrm{erg} \mathrm{cm}^{-2} \mathrm{~s}^{-1} \mathrm{sr}^{-1} \AA^{-1}\right]$

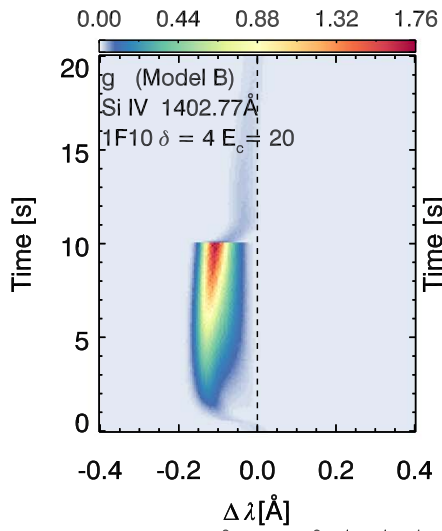

Intensity $\left[\times 10^{8} \mathrm{erg} \mathrm{cm}^{-2} \mathrm{~s}^{-1} \mathrm{sr}^{-1} \AA^{-1}\right]$

$\begin{array}{lllll}0.000 & 0.286 & 0.572 & 0.858 & 1.144\end{array}$

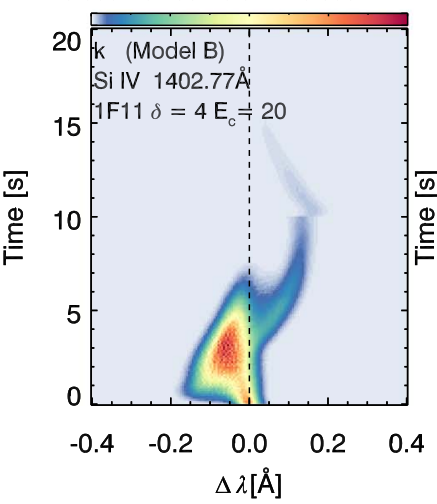

$\begin{array}{lllll}0.0 & 1.1 & 2.2 & 3.3 & 4.4\end{array}$

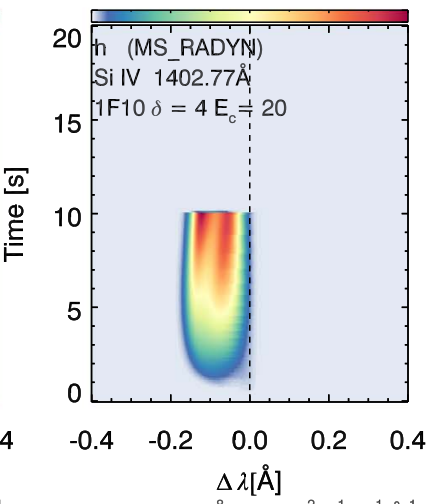

Intensity $\left[\times 10^{8} \mathrm{erg} \mathrm{cm}^{-2} \mathrm{~s}^{-1} \mathrm{sr}^{-1} \AA^{-1}\right]$

$\begin{array}{lllll}0.00 & 1.94 & 3.88 & 5.82 & 7.77\end{array}$

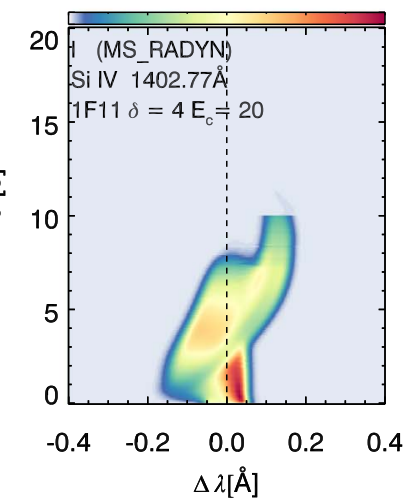

Figure 4. Si IV $\lambda 1402.77$ line profiles in the first $20 \mathrm{~s}$ of the flare simulations computed using Model B (panels (a), (c), (e), (g), (i), (k)) and computed using MS_RADYN (panels (b), (d), (f), (h), (j), (l)). Six flare simulations are shown. Each has nonthermal electron distribution parameters of $\delta=4$ and $E_{c}=20 \mathrm{keV}$, with the injected energy flux varying for each pair of images: 5F8 (panels (a), (b)), 1F9 (panels (c), (d)), 5F9 (panels (e), (f)), 1F10 (panels (g), (h)), 5F10 (panels (i), (j)), and 1 F11 (panels (k), (1)). Within each image the rows are different times in the simulation, so that the profiles are shown as wavelength vs. time. Note that the scale is different for each panel, indicated in the relevant color bars. For simulations with very intense emission in the heating phase, the cooling phase is not visible owing to scaling.

The resonance line ratios differ significantly between the two models, acutely in the stronger flares. When computed via Model $B$, the ratio is very close to 2 , as expected in the optically thin case, though with some deviations during the flare. This is likely caused by a temperature dependence of the line ratio that is present when the lines form in cooler plasma. When computed by MS_RADYN, there is significant deviation, with values as low as $R_{\text {res }} \approx 1.84$ and as high as $R_{\text {res }} \approx 2.27$. This implies that optical depth effects are substantial at these times. As discussed by Rathore \& Carlsson (2015), the intensity ratio can in effect take any value and represents the ratio of the source functions of the line. The magnitudes of the source functions depend on where they thermalize in the atmosphere. Since Si IV $\lambda 1393.75$ has twice the opacity of Si IV $\lambda 1402.77$ (proportional to the ratio of oscillator strengths of the lines, since they share a common lower level, and $\chi_{\nu} \propto n_{1} f_{\mathrm{lu}}$ ), it thermalizes higher in altitude where temperatures are larger. While the $\operatorname{Mg}$ II $h$ and $k$ lines 

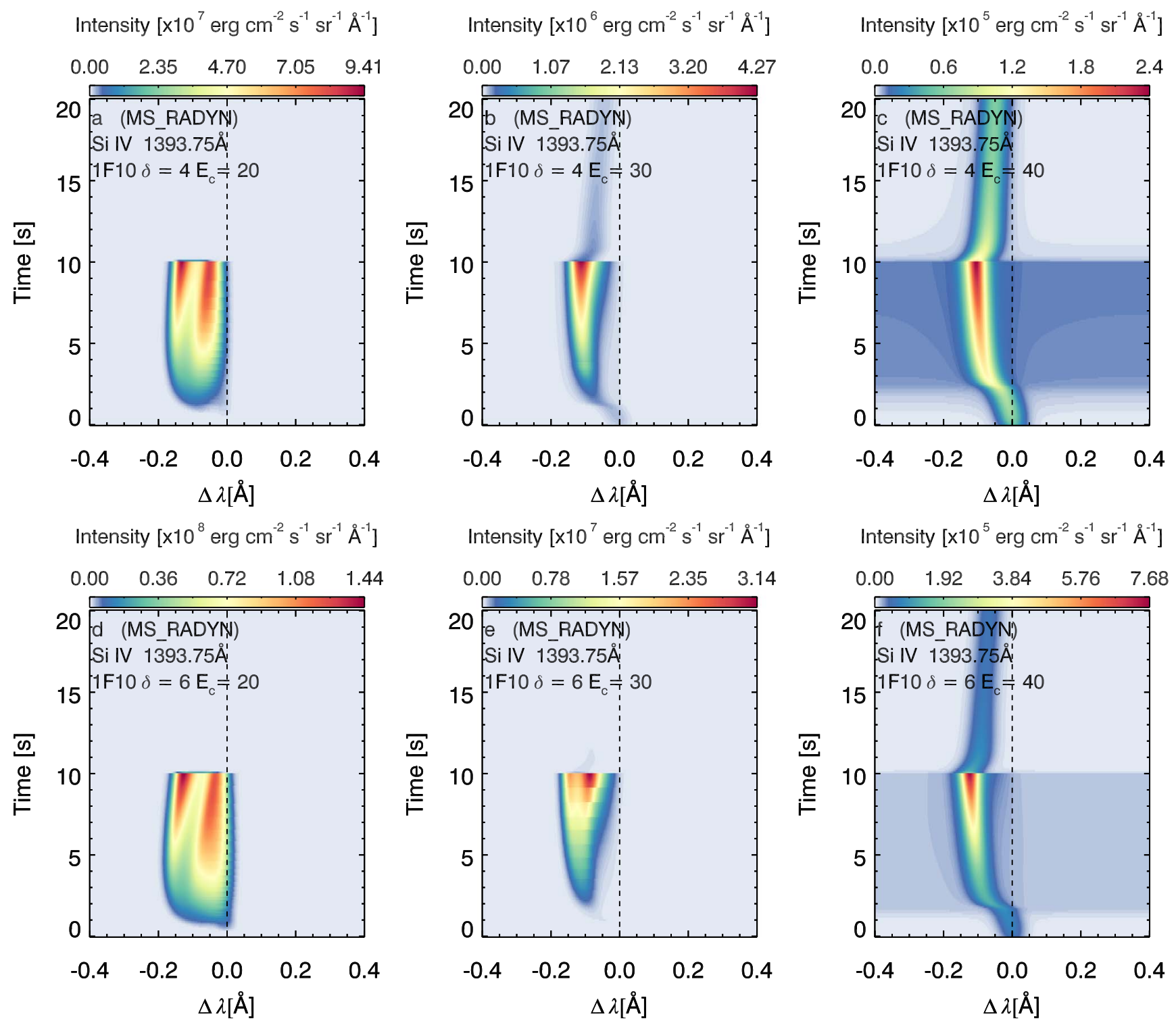

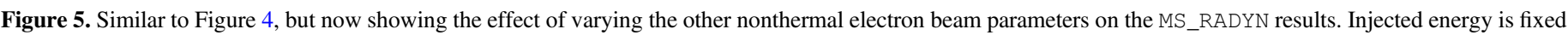

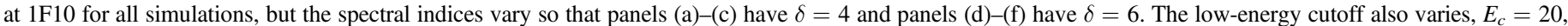
30 , and $40 \mathrm{keV}$ from left to right in each row.

typically have a ratio much lower than their optically thin limit both in the quiet Sun and in flares (Leenaarts et al. 2013; Kerr et al. 2015), the Si IV resonance line intensity ratio is more variable and can exceed the optically thin limit. We speculate that this is due to the Si IV lines being less optically thick than $\mathrm{Mg}$ II $h$ and $k$ and that the $\lambda 1393.75$ line appears to become affected by opacity more than the $\lambda 1402.77$ line. A detailed investigation of the formation properties will elucidate, and this will be the focus of a subsequent investigation.

\subsection{Line Formation}

\subsubsection{Contribution Functions}

A useful way to understand the complex spectral line formation under optically thick conditions is to study the contribution functions to the emergent intensity, $C_{\nu \mu}$ (Magain 1986; Carlsson 1998). This effectively shows where in the atmosphere emission at frequency $\nu$ is formed. Integrating $C_{\nu \mu}$ through a depth scale yields the emergent intensity $I_{\nu \mu}$. Using the height $z$ as the depth scale, then the emergent intensity is

$$
I_{\nu \mu}=\int C_{\nu \mu}(z) d z=\int \frac{1}{\mu} \chi_{\nu}(z) S(z) e^{-\tau_{\nu}(z) / \mu} d z,
$$

where $\chi_{\nu}(z)$ is the monochromatic opacity, $S(z)$ is the frequency-independent source function (since we use the assumption of CRD), $\tau_{\nu}(z)=\int \chi_{\nu} d z$ is the optical depth, and $\mu=\cos \theta$ is the viewing angle relative to the normal. We use $\mu \approx 0.953$ throughout this work, which is near disk center, and drop the $\mu$ subscript.

For each MS_RADYN simulation $C_{\nu}(z)$ was computed for the resonance lines at 1393.75 and $1402.77 \AA$ and the height of optical depth unity $\left(\tau_{\lambda}=1\right)$ compared to the locations of strong emission. If $C_{\nu}(z)$ originates near the height of $\tau_{\lambda}=1$, then 

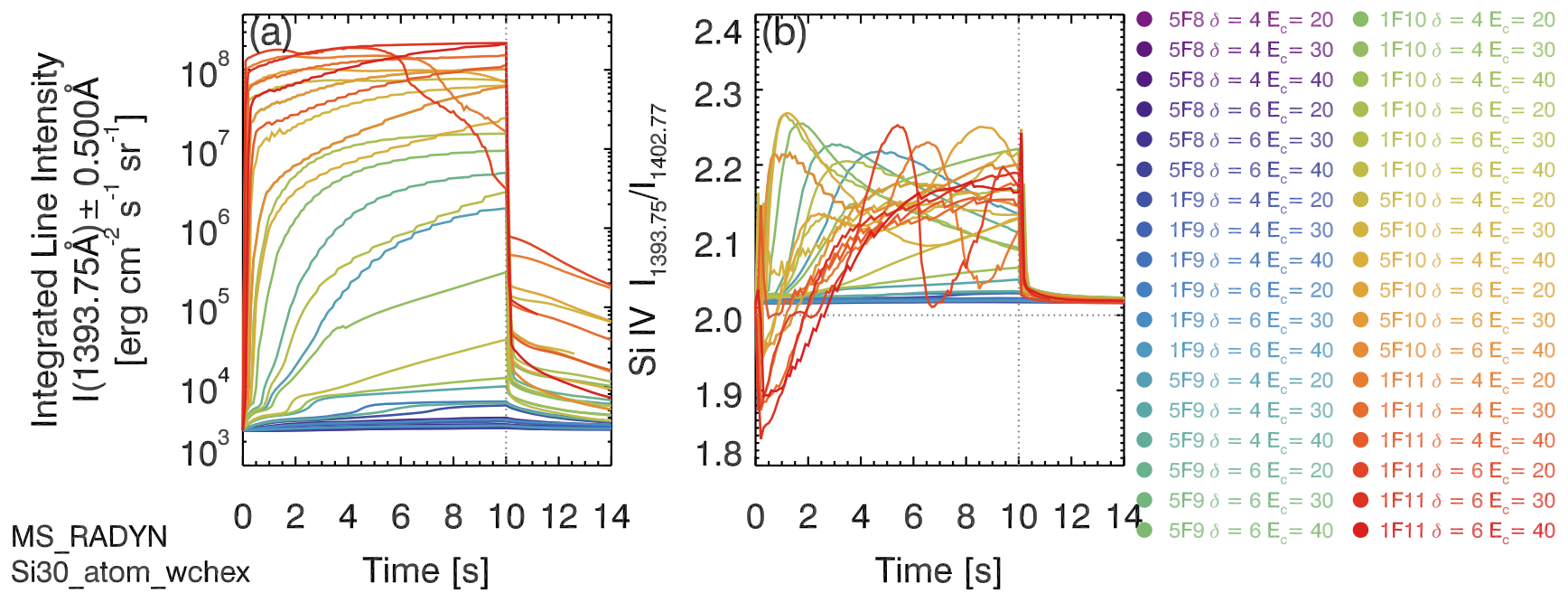

Figure 6. Panel (a) shows light curves of the integrated intensity ( $\lambda_{\text {rest }} \pm 0.5 \AA$ ) of the Si IV $\lambda 1393.75$, as computed by MS_RADYN, for the first $t=14 \mathrm{~s}$ of the flare simulations (recall that the heating phase lasts $t=10 \mathrm{~s}$, indicated by the dotted line). Panel (b) shows the ratio $R_{\text {res }}=I_{1393} / I_{1402}$ over the same duration. In both panels color represents the different simulations.

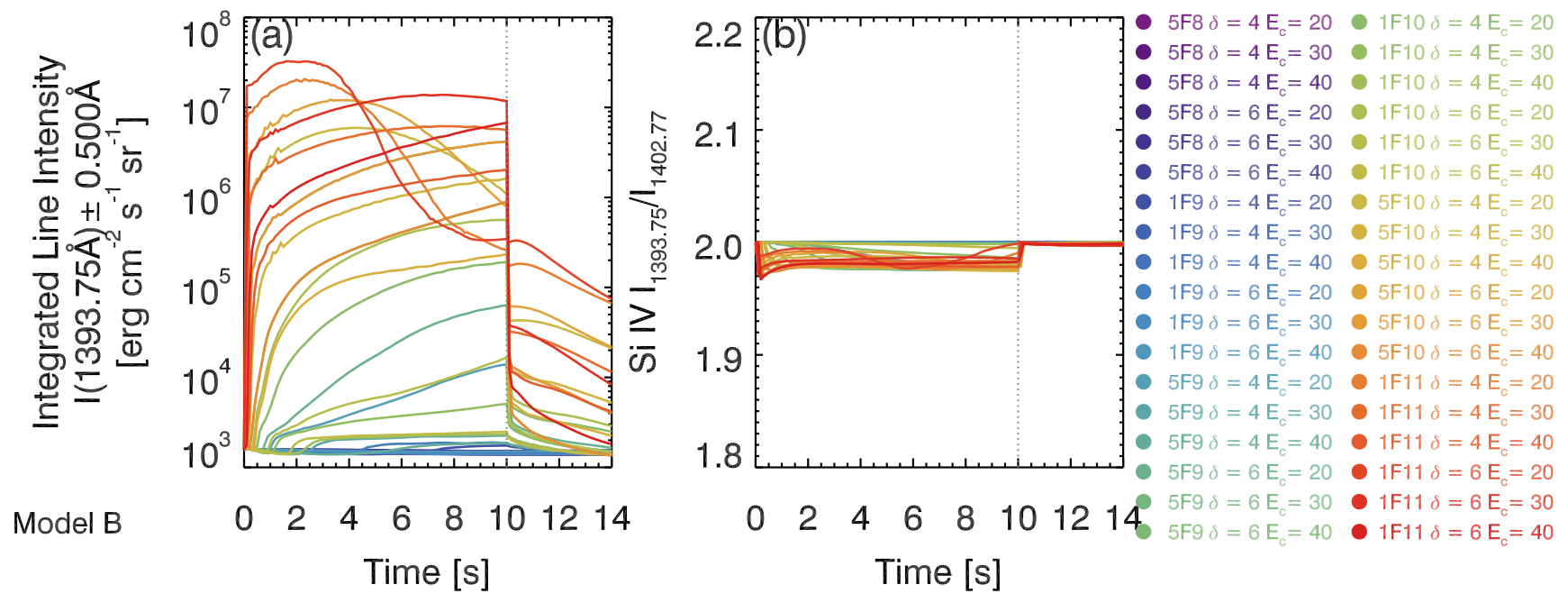

Figure 7. Same as Figure 6, but showing results as computed using the optically thin assumption via Model B.

optical depth effects are important for line formation. If emission originates sufficiently far from the height of $\tau_{\lambda}=1$, then the line can be considered optically thin. In some cases the emergent line profile can be a combination of emission produced where opacity effects are considerable and optically thin emission. Opacity effects were time dependent in some simulations, with the importance varying over time, as a function of the atmospheric structure. In some cases the optical depth at the formation height did not reach unity but was still non-negligible $\left(\tau_{\lambda} \geqslant 0.1\right)$. Finally, the optical depth effects were wavelength dependent, such that the width of the line that was affected by opacity varied. In the atmospheres where the Si IV fraction significantly increased, a large part of the line was affected by opacity, while in atmospheres with more modest increases only a narrow region around the line core became optically thick (where the absorption profile is maximum).

Optical depth effects were present for all simulations in which the injected energy flux was equal to $5 \mathrm{~F} 10$ or greater. For smaller magnitudes of injected energy flux the presence of optical depth effects depends on the other parameters of the nonthermal electron beams. Sufficient energy must be deposited in the upper chromosphere to result in an extended high-temperature region over which the SiIV population becomes enhanced.

The location of energy deposition is a function of both the power-law (spectral) index $\delta$ and the low-energy cutoff of the distribution $E_{c}$. A larger value of $\delta$ means that there are relatively more lower-energy electrons than higher-energy electrons (if all parameters are fixed), which are thermalized at lower column depths. The distribution contains nonthermal electrons with energies as low as $E_{c}$ so that a smaller value of $E_{c}$ for a fixed energy flux means that more power is carried by the lower-energy electrons, resulting in greater heating at low column depth. We show in Table 1 in which simulations optical depth effects were present. Note that the length of time over which optical depth effects were present varied in each simulation (though for the high-energy simulations they typically lasted for the entire duration of the heating phase), so we just indicate in Table 1 if they were present at some point during the flare. If charge exchange processes are excluded, 
Table 1

Presence of Si IV Resonance Line Optical Depth Effects in the MS_RADYN Flare Simulations Using Si30_atom_wchex

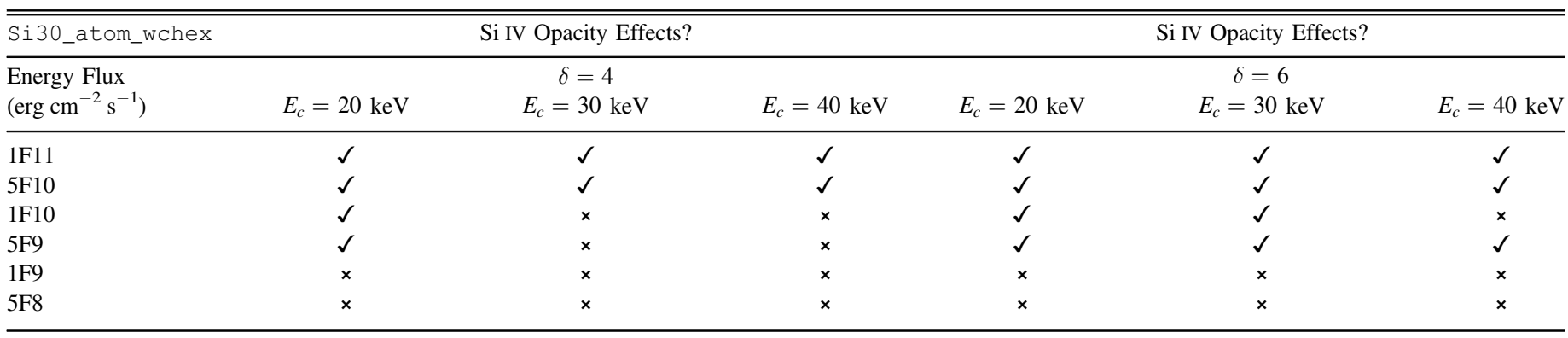

Note. The duration and strength of optical depth effects vary in each simulation; we are just indicating here whether they are non-negligible at some point during the flare.

Table 2

Presence of Si IV Resonance Line Optical Depth Effects in the MS_RADYN Flare Simulations Using Si30_atom

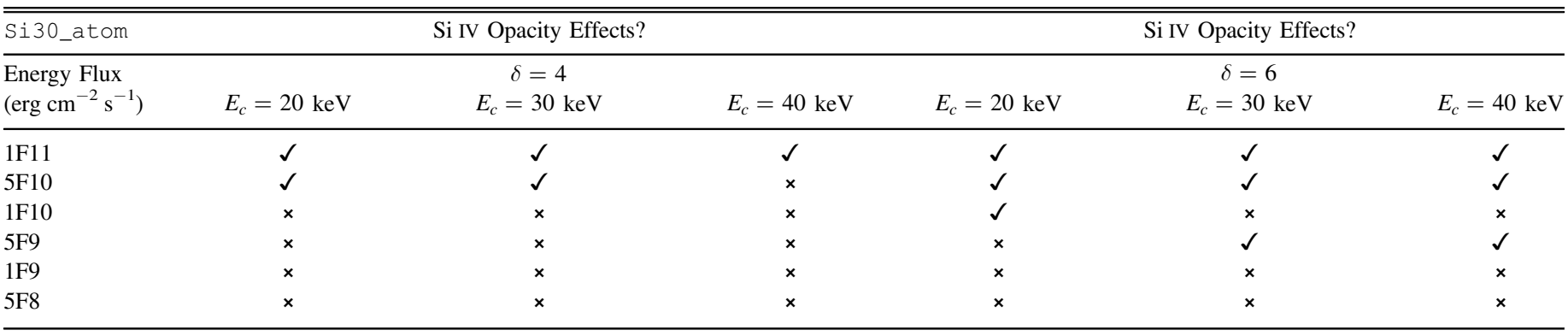

then there is less Si IV present at lower temperatures and consequently a smaller opacity. Table 2 indicates that by excluding charge exchange SiIV formation is affected by opacity in fewer cases.

To illustrate the formation of the line in various simulations, we show examples of the contribution function to the emergent intensity in Figures 8 and 9. Each of the panels indicates the rest wavelength of the line core in the upper right corner, the time in the simulation in the upper left corner, and the injected electron beam parameters in the lower left corner. The background image in color is the contribution function, where red is more intense and blue/white is less intense. We have normalized the contribution function within each wavelength bin in order to bring out weaker details in the line wings, which would be washed out by the much more intense line core. The line core formation region is typically very narrow compared to the line wings. Both the core and wings can be combinations of emission formed near the $\tau_{\lambda}=1$ layer and higher-lying optically thin regions. The contribution functions are shown as functions of height (left axis) and Doppler shift (bottom axis), with the corresponding wavelength shift from line center on the top axis. On the same scale the height at which $\tau_{\lambda}=1$ is shown as a black solid line. The atmospheric velocity as a function of height is shown as a light-gray dashed line. Upflows and blueshifts are negative; downflows and redshifts are positive. Finally, the line profiles in units of radiation temperature (right axis) are shown as dark-gray solid lines.

Figure 8 shows the effect of varying the injected energy flux on the formation of the $\lambda 1402.77$ line. Each of the simulated values of injected energy flux for fixed $\delta=4$ and $E_{c}=20 \mathrm{keV}$ is shown at $t=5 \mathrm{~s}$. For the weakest flare simulations (5F8 and
1F9) the point of optical depth unity is not reached until deep into the atmosphere at $z \approx 0.4 \mathrm{Mm}$. The line forms in a vanishingly thin layer of the TR, around $z \approx 1.2 \mathrm{Mm}$. The lines can be considered to form under optically thin conditions. In panel (b) there is a Doppler shift of the line since it forms in an upflowing region moving at $v \approx 10-15 \mathrm{~km} \mathrm{~s}^{-1}$.

In the higher-energy simulations opacity effects are present, to varying degrees. Panel (c) shows the 5F9 simulation, where the core of the line reaches optical depth unity at a much higher altitude than in the weakest simulations, around $z \approx 1.2 \mathrm{Mm}$, where that part of the line forms. The line wings are again optically thin. Hence, the line core is optically thick and blueshifted, but there is a red wing asymmetry caused by an optically thin component that forms over an extended height range along a velocity gradient. If the energy is increased (panel (d)), then the contribution function appears similar, but now the range of wavelengths that form under optically thick conditions has increased so that the line core is wider. In this case the velocity gradient produces an optically thick component redward of the line core, increasing the width of the red emission peak relative to the blue. In the strongest flares (panels (e), (f)) the dynamics of the atmosphere are more dramatic such that the velocity structure is more complex. Here there are sharper gradients, and strong condensations begin to form. Multiple optically thick components begin to form at different heights, and the profiles can appear more complicated.

Figure 9 shows the effects of varying the other beam parameters, which effectively is changing the location in the atmosphere at which the energy is injected. The resulting atmospheric structure can vary sufficiently given the location of energy injection so that Si IV formation is very different even 

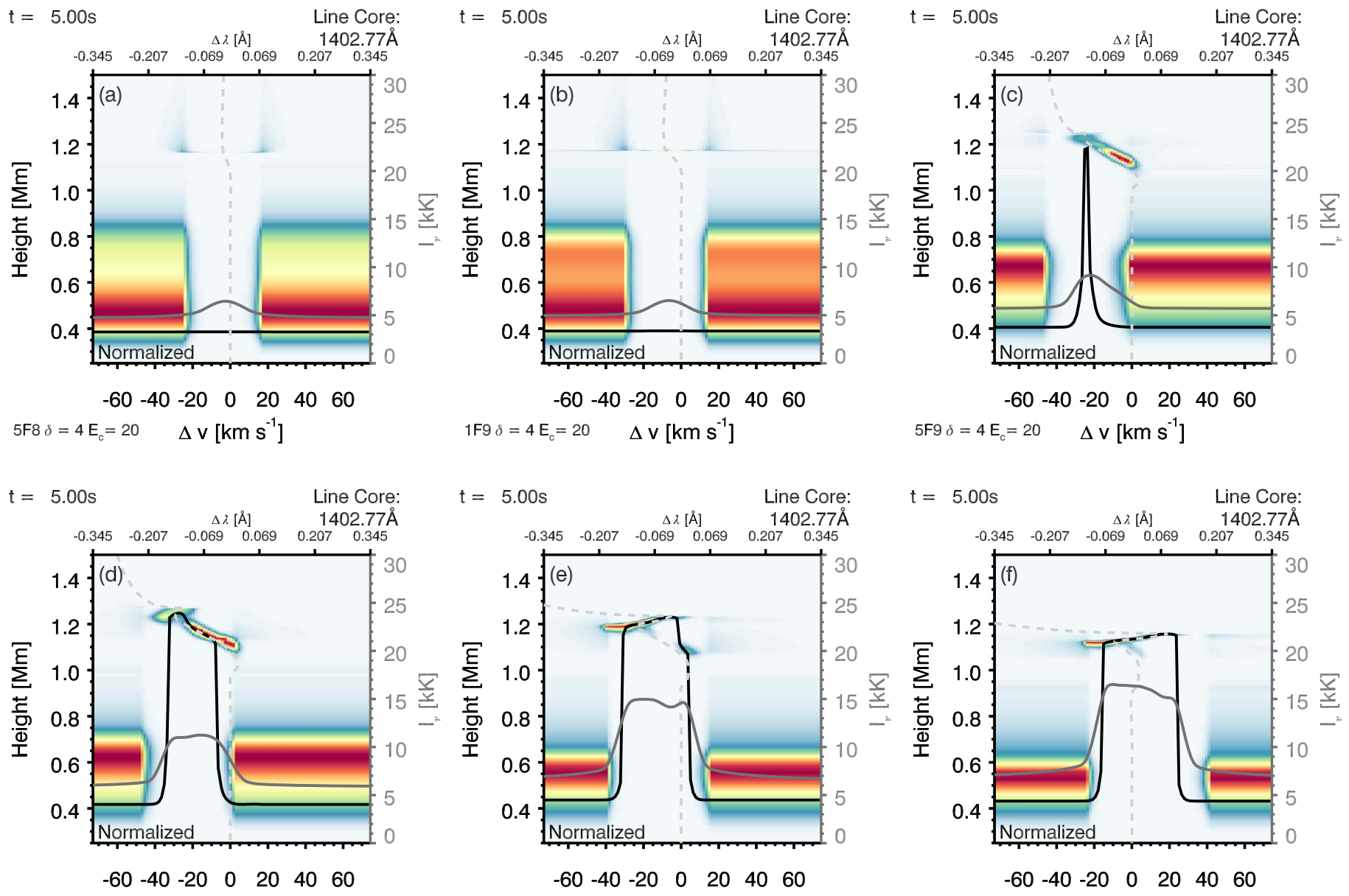

$1 \mathrm{~F} 10 \delta=4 \mathrm{E}_{\mathrm{c}}=20 \quad \Delta \mathrm{v}\left[\mathrm{km} \mathrm{s}^{-1}\right]$

$5 \mathrm{~F} 10 \delta=4 \mathrm{E}_{\mathrm{c}}=20 \quad \Delta \mathrm{v}\left[\mathrm{km} \mathrm{s}^{-1}\right]$

$1 \mathrm{~F} 11 \delta=4 \mathrm{E}_{\mathrm{c}}=20 \quad \Delta \mathrm{v}\left[\mathrm{km} \mathrm{s}^{-1}\right]$

Figure 8. Panels illustrating the formation of Si IV $\lambda 1402.77$ at $t=5 \mathrm{~s}$ in six MS_RADYN simulations with varying injected energy flux. The spectral index and low-energy cutoff of the electron beam are $\delta=4$ and $E_{c}=20 \mathrm{keV}$, with the injected energy flux ranging from panel (a) to panel (f): $F=[5 \mathrm{~F} 8,1 \mathrm{~F} 9,5 \mathrm{~F} 9$, $1 \mathrm{~F} 10,5 \mathrm{~F} 10,1 \mathrm{~F} 11] \mathrm{erg} \mathrm{cm}^{-2} \mathrm{~s}^{-1}$. The background image is the contribution function to the emergent intensity defined on height (left axis), Doppler velocity (bottom axis), and wavelength scales (top scale), where red is more intense. The contribution function has been normalized within each wavelength bin to bring out details in the line wings. On each panel is the atmospheric velocity as a function of height (light-gray dashed line), the height at which optical depth unity $\left(\tau_{\lambda}=1\right)$ is reached (black solid line), and the line profile in units of radiation temperature (right axis; dark-gray solid line).

for a fixed amount of injected energy (in these cases we show 1F10). The top panels have $\delta=4$ and $E_{c}=20,30,40 \mathrm{keV}$ (panels (a)-(c), respectively), and the bottom panels have $\delta=6$ and $E_{c}=20,30,40 \mathrm{keV}$ (panels (d)-(f), respectively). It is immediately clear that the cases of larger values of $E_{c}$ are more likely to lead to optically thin emission, whereas depositing more energy at higher altitudes can lead to optical depth effects. Similarly, comparing panels (b) and (e) shows that a harder spectrum $(\delta=4$, meaning that more energy is carried by deeply penetrating electrons) has a similar effect.

\subsubsection{Plasma Properties at the Height of Peak Emission}

During the flares, opacity effects become important for the resonance line photons when the column density of Si IV through which they have to travel becomes sufficiently large. However, it is not possible to obtain this quantity before running a simulation, nor is it easy to obtain this from observations. It is instructive to turn to another metric of the plasma that might serve as a proxy and inform under what conditions optical depth becomes significant. If the Si IV ground-state population is sufficiently high, then the atmosphere may be optically thick to those wavelengths. In relative terms, this can happen if the Si IV populations are modest over a large geometric region, or if they are very high over a short geometric region. Integrating the mass density over the rough formation temperature range of Si IV can provide a proxy of the amount of Si IV a photon must travel through and a threshold of at what point the atmosphere becomes opaque to the Si IV resonance lines. That is, the column mass over the formation region of Si IV must be at a certain value.

The temperature range at which we evaluated this threshold was $40 \mathrm{kK} \leqslant T \leqslant 100 \mathrm{kK}$. The cumulative sum $m_{\mathrm{col}}$, $\sum_{z=z_{100 \mathrm{~K}}}^{z_{40 \mathrm{~K}}} \rho(z) \delta z$, starting at the height at which $T=100 \mathrm{kK}$, $z_{100 \mathrm{kK}}$, until the height at which $T=40 \mathrm{kK}, z_{40 \mathrm{kK}}$ (where $\rho$ is the mass density). These temperatures represent typical formation temperature ranges, but we note that during the flare the lines can occasionally form outside this range. For this initial study, though, we deemed it a suitable choice to provide insight into the atmospheric properties where Si IV forms. Since the temperature structure can be complex with local minima and maxima, we selected the minimum height at which $T \geqslant 40 \mathrm{kK}$ and the maximum height at which $T \leqslant 100 \mathrm{kK}$. The mass density was then interpolated to a much finer grid with 1000 cells, and the sum was performed. 

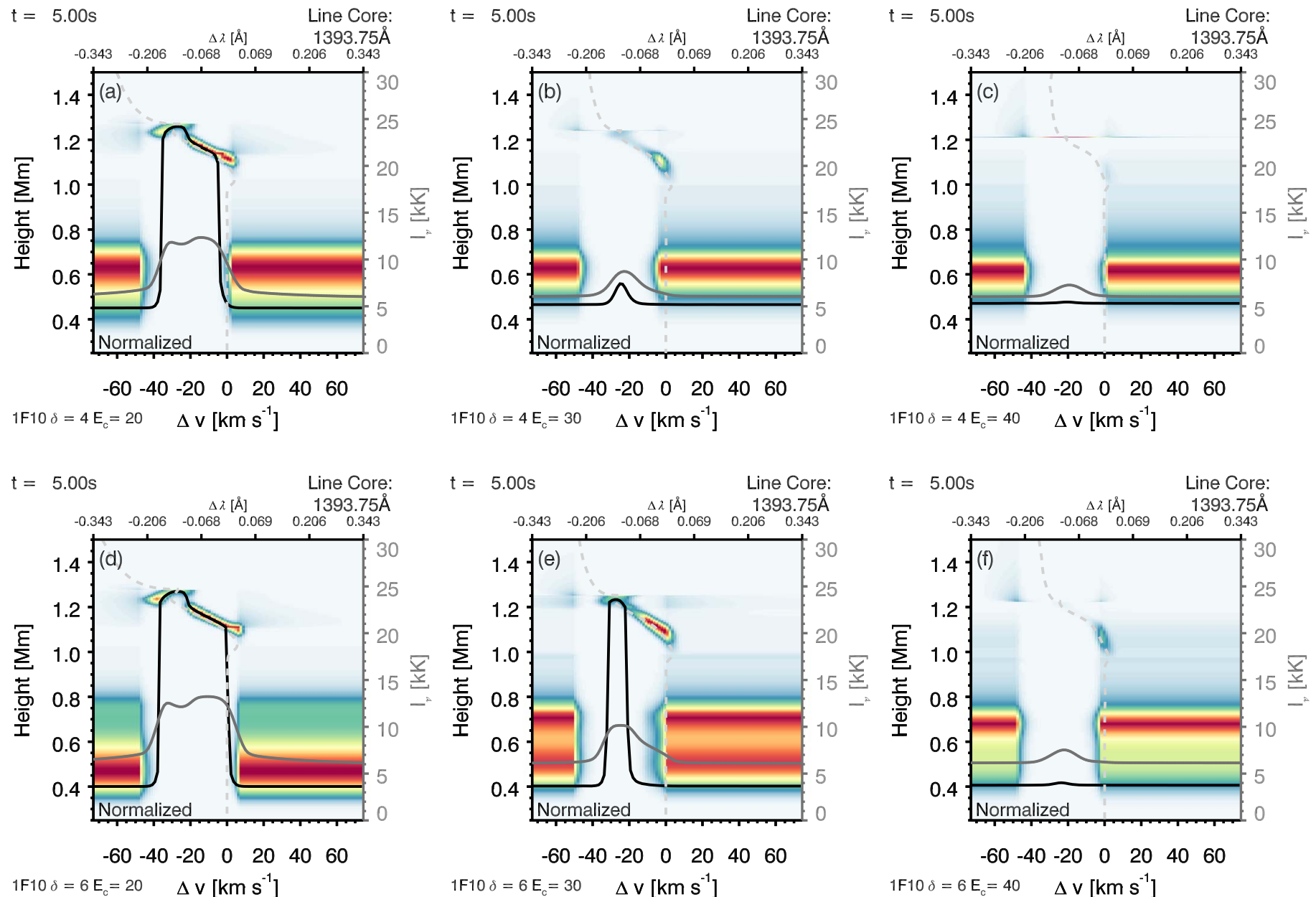

$1 \mathrm{~F} 10 \delta=6 \mathrm{E}_{\mathrm{c}}=20 \quad \Delta \mathrm{V}\left[\mathrm{km} \mathrm{s}^{-1}\right]$

$1 \mathrm{~F} 10 \delta=6 \mathrm{E}_{\mathrm{c}}=30 \quad \Delta \mathrm{v}\left[\mathrm{km} \mathrm{s}^{-1}\right]$

$1 \mathrm{~F} 10 \delta=6 \mathrm{E}_{\mathrm{c}}=40 \quad \Delta \mathrm{v}\left[\mathrm{km} \mathrm{s}^{-1}\right]$

Figure 9. Panels illustrating the formation of Si IV $\lambda 1393.75$ at $t=3 \mathrm{~s}$ in six MS RADYN simulations with varying constant injected energy flux of $1 \mathrm{~F} 10 \mathrm{erg} \mathrm{cm}^{-2} \mathrm{~s}^{-1}$. The spectral index of the top row is $\delta=4$, and the bottom row is $\delta=6$. For each row the low-energy cutoff varies from left to right with values of $E_{c}=[20,30,40] \mathrm{keV}$. The panels are as described in Figure 8.

Comparing $m_{\text {col }}$ to the optical depth can allow a rough threshold to be identified, above which the lines are likely to be optically thick. For simulations that produce a sufficiently high $m_{\text {col }}$ the full NLTE radiation transfer should be solved for Si IV, and for simulations in which $m_{\mathrm{col}}$ is low enough, a process similar to Model B can be used (with the caveat that the intensities will be different by some amount owing to exclusion of charge exchange). Observationally this is not a straightforward metric to measure, but we can use our model grids to determine whether there is a parameter range for the nonthermal electron distribution that typically results in the atmosphere exceeding this threshold. Hard X-ray observations from the RHESSI (Lin et al. 2002) or Fermi (Meegan et al. 2009) satellite, or from other observatories, can be used to derive parameters for the nonthermal electron distribution, which can be compared to our study, providing some guidance on the likelihood that opacity effects are present.

For each snapshot output from MS_RADYN the wavelength at which the intensity of the resonance lines peaked was selected. The temperature and optical depth at the height of the peak of the contribution function for that wavelength were recorded. The heights corresponding to the FWHM of the contribution function and the temperatures and optical depths at those heights were also recorded. Choosing the peak line intensity helped to avoid the requirement of tracking the line core position (which becomes ambiguous for optically thick lines anyway) in atmospheres with strong flows. In the optically thin cases the line core and position of peak line intensity are typically the same. In the optically thick cases with self-absorption features, the peak line intensity is a component of the line that forms somewhat below the core.

Figure 10 shows the gas temperature as a function of the optical depth at the formation height of the line peak at $t=0 \mathrm{~s}$ in panel (a), and $m_{\mathrm{col}}$ also as a function of optical depth in panel (b), at $t=0 \mathrm{~s}$. The error bars represent the same values at the heights of the FWHM of the contribution function. Figures 10 (c)-(h) show the same properties at $t=3.5 \mathrm{~s}, t=7.5 \mathrm{~s}$, and $t=15 \mathrm{~s}$ respectively, illustrating the progression during the flare simulations. These figures show results derived from the $\lambda 1393.75$ line only. The $\lambda 1402.77$ line produced similar results, but with optical depths that were sometimes smaller than the $\lambda 1393.75$ line.

At $t=0 \mathrm{~s}$ the temperature of line peak formation was $\log T \approx 4.82(T \approx 65.9 \mathrm{kK})$. The optical depth at the formation height was $\tau_{\lambda}=0.018$. For reference, excluding charge change meant that the line peak formed at $\log T \approx 4.87(T \approx 74.2 \mathrm{kK})$, with an optical depth $\tau_{\lambda}=0.014$. 

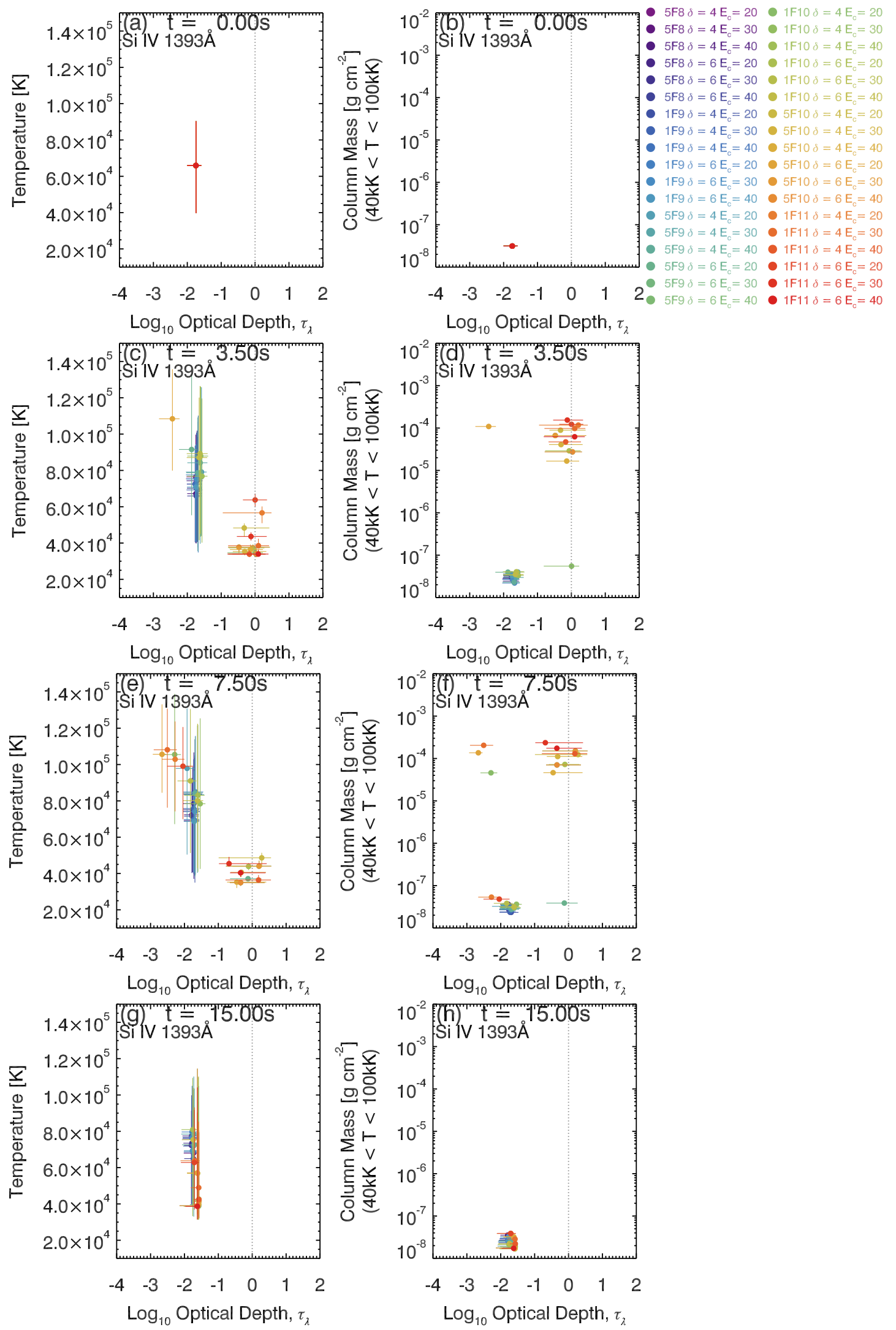

Figure 10. Plasma properties at $t=0,3.5,7.5,15 \mathrm{~s}$ for the $\lambda 1393.75$ line, from the MS_RADYN simulations. (a) Temperature at the altitude at which the contribution function to the emergent intensity at peak line intensity is maximum, as a function of optical depth at that altitude (at the wavelength of line peak intensity). Error bars show temperature and optical depth at heights at which the contribution function reaches FWHM. (b) Column mass, $m_{\text {col }}$, between $40 \mathrm{kK} \leqslant T \leqslant 100 \mathrm{kK}$ as a function of optical depth at the formation height. In both panels the colors refer to different nonthermal electron distribution parameters as indicated in the annotation. The dotted line shows $\tau_{\lambda}=1$. 


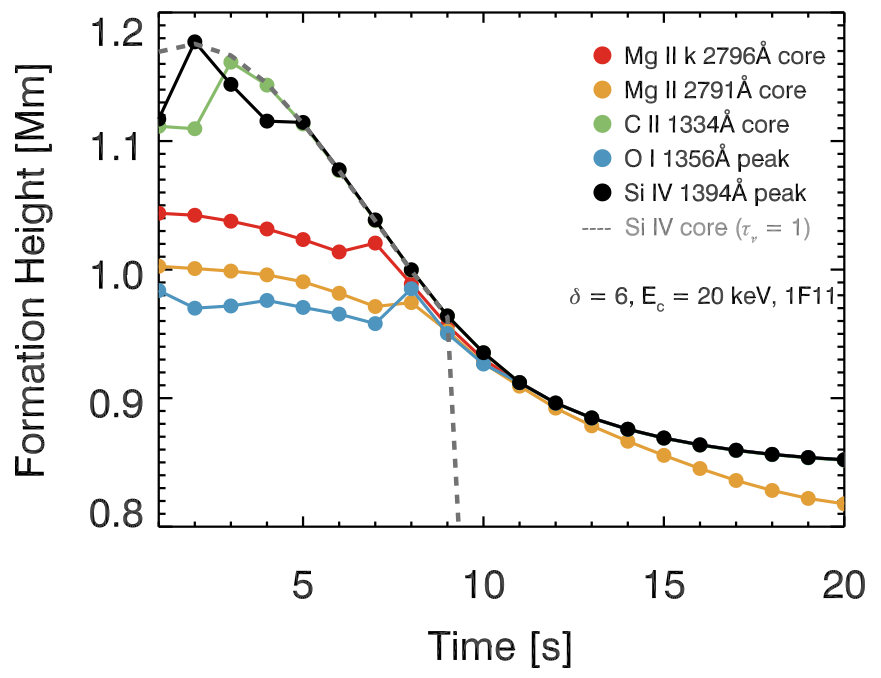

Figure 11. Formation heights of the Si IV $\lambda 1393.75$ core (gray dashed line), Si IV $\lambda 1393.75$ emission peak (black circles), C II $\lambda 1334$ core (green circles), $\mathrm{Mg}$ II $k$ core (red circles), Mg II $\lambda 2791$ core (orange circles), and O I $\lambda 1356$ emission peak (blue circles) as functions of time in the $1 \mathrm{~F} 11 \delta 6 E_{c} 20$ simulation. Note that in the gradual phase most lines (apart from Mg II $\lambda 2791$ ) form in a very narrow region (underneath the black symbols).

From these figures it is clear that in many of the simulations the flare environment is such that opacity effects are significant for the Si IV resonance line profiles, forming in an optically thick regime (with $\tau_{\lambda} \gtrsim 0.5$ ). Overall, two populations exist, one where opacity effects are important and one in which they are not. In the latter, the lines generally form in hotter plasma, with $T \approx 60-100 \mathrm{kK}$, with values of $m_{\mathrm{col}} \approx 10^{-8} \mathrm{~g} \mathrm{~cm}^{-2}$. Here any increase of the Si IV fraction is small so that the opacity change (if any) is also small. The plasma is optically thin to the resonance lines.

In the other population the lines form at somewhat cooler temperatures, with $T \approx 30-60 \mathrm{kK}$, and over larger column masses on the order of $m_{\text {col }} \gtrsim 5 \times 10^{-6} \mathrm{~g} \mathrm{~cm}^{-2}$ to $m_{\text {col }} \sim 5 \times$ $10^{-4} \mathrm{~g} \mathrm{~cm}^{-2}$, so that the opacity increase is significant. Over time, as $m_{\text {col }}$ increased, there was a transition from $\tau_{\lambda} \ll 1$ to $\tau_{\lambda} \sim 1$, with a rough threshold of $m_{\text {col }} \approx 5 \times 10^{-6} \mathrm{~g} \mathrm{~cm}^{-2}$ required for the plasma to be optically thick to the Si IV resonance lines.

The dynamics of the atmosphere are time dependent, so that some of the simulations take time to reach the $m_{\text {col }}$ threshold and transition from being optically thin to optically thick. The atmosphere can become compressed, reducing the geometric size of the region exhibiting temperature and electron density enhancements. The path length through which photons must pass is consequently reduced. This process occurs more rapidly when heating is concentrated higher in the atmosphere. The resonance lines can transition back to being optically thin before the end of the heating phase, for example, $1 \mathrm{~F} 11 \delta 6 E_{c} 20$ in Figures 10(e) and (f). After the heating phase, the temperature and electron density both quickly decrease, and in all simulations the lines (while still at a greater intensity that their quiet-Sun values) are optically thin.

Our use of $m_{\text {col }}$ between $40 \mathrm{kK} \leqslant T \leqslant 100 \mathrm{kK}$ as a proxy of optical depth is, of course, not a perfect rule, and formation temperatures can fall outside of the bounds we have selected.
This leads to outliers from the general trend. Outliers are also possible owing to the selection of the peak intensity, as other line components (e.g., a centrally reversed line core) may be optically thick even if the peak intensity is not. However, we believe that this is a robust enough measure for this initial study, providing a proxy for when opacity effects are substantial in many of the simulations.

\subsection{Formation Height relative to Other IRIS Spectral Lines}

Using $1 \mathrm{~F} 11 \delta 6 E_{c} 20$ as a case study, we compare the height of formation of the Si IV emission to the formation height of other strong spectral lines observed by IRIS in flares. Following the approach described in Kerr et al. (2016) and Kerr (2017), snapshots of the RADYN atmospheres were used as input to the $\mathrm{RH}$ radiation transfer code (Uitenbroek 2001) to obtain synthetic spectra of the $\mathrm{Mg}$ II $k$ line, the $\mathrm{Mg}$ II $\lambda 2791$ line, the C II $\lambda 1334$ line, and the O I $\lambda 1356$ line. The RH code was required here because these ions are not simulated by RADYN, and the $\mathrm{Mg}$ II $k$ line requires a PRD treatment. A caveat here is that $\mathrm{RH}$ assumes statistical equilibrium, which is partly mitigated by using the nonequilibrium electron density from RADYN. Future efforts will attempt to simulate these lines using MS_RADYN, investigating nonequilibrium effects. We use a modified version of $\mathrm{RH}$ in which the hydrogen populations as input from RADYN (and thus were the nonequilibrium populations) were held constant while still solving the radiation transfer. Populations of the other elements were allowed to iterate. This means that the computation of the hydrogen opacity (a significant source of background opacity) and the effects of charge exchange, which are important for O I $\lambda 1356$ formation (Lin \& Carlsson 2015), used the more accurate hydrogen populations from RADYN.

For optically thick lines (the $\mathrm{Mg}$ II $k, \mathrm{Mg}$ II $\lambda 2791$, and C II $\lambda 1334$ lines) we define the formation height of the line core to be the maximum height of the $\tau_{\lambda}=1$ surface. These heights are shown in Figure 11 . The $\mathrm{OI} \lambda 1356$ line is optically thin throughout the flare, and so we instead show the height at which the contribution function to the emergent intensity is largest, for the wavelength corresponding to the peak line intensity.

Since the Si IV $\lambda 1393.75$ line transitions between optically thick and optically thin, we show both the height at which $\tau_{\lambda}=1$ is maximized and the height at which the contribution function to the emergent intensity is largest, for the wavelength corresponding to the peak line intensity. The former is shown as a dashed gray line, and the latter as black circles. When the line is optically thick, the $\tau_{\lambda}=1$ represents the line core, and the latter metric represents the formation height of the emission peak. When the line forms in optically thin conditions, the $\tau_{\lambda}=1$ surface does not hold any special meaning, as the intensity originates far from this height, but we include it in Figure 11 to illustrate the transition. These two measures both show similar heights during the heating phase when the line is optically thick, particularly when the chromosphere compresses so that the formation height difference of the line core and emission peak is small. Shortly before $t=10 \mathrm{~s}$, the height of the $\tau_{\lambda}=1$ surface drops, whereas the line continues to form in the upper chromosphere/TR. At these times the $\tau_{\lambda}=1$ no longer represents the line core, and the line is considered optically thin. 
There is a stratification in the heights at which the various lines form, which varies with time in the flare. Initially the core of the Si IV $\lambda 1393.75$ line forms highest in altitude, followed by the $\mathrm{C}$ II resonance lines, the emission peak of the Si IV $\lambda 1393.75$ line (recall that the line has a self-absorption feature that is the core), the $\mathrm{Mg}$ II resonance lines, the $\mathrm{Mg}$ II subordinate lines, and finally the O I $\lambda 1356$ line. After several seconds, mass motions, and the changing temperature and density structure, raise the height of the C II resonance lines and reduce the height difference between the Si IV resonance line core and the emission peak. Toward the end of the heating phase the compression of the chromosphere results in the lines forming in a very narrow region, which persists into the cooling phase. The evolution of the stratification of line components will be examined in more detail, in order to identify potential diagnostics such as temperature and velocity gradients.

\section{Summary and Conclusions}

The flare response of the Si IV resonance lines was computed under the assumption that they are formed in optically thin conditions using atomic data from the CHIANTI database (Model B) and using full NLTE, nonequilibrium radiation transfer via the minority species version of the RADYN radiation hydrodynamics code (MS_RADYN). In both models we used 36 flare simulations computed via RADYN, which covered a wide range of parameter space of nonthermal electron beams. The initial results from this model grid were presented, illustrating the changes in intensities, shapes, and ratios, as well as some basic formation properties. The Si IV lines were also placed in context with other strong lines observed by the IRIS spacecraft in a case study of one simulation.

Generally, weaker flares resulted in reasonably similar Si IV line emission when using either Model B or MS_RADYN. In stronger flares the lines differed, sometimes significantly, in terms of intensity, the presence of self-absorption features, asymmetries, Doppler motions, and widths. The intensity ratio of the resonance lines when computed by MS_RADYN deviates from the optically thin limit of 2 , in the stronger flares.

Our results suggest that in flaring conditions, or possibly during smaller-scale but intense heating events, the populations of Si IV can become substantially enhanced over a large enough geometric region so as to result in the upper chromosphere and lower TR becoming (semi)opaque to $\mathrm{Si}$ IV resonance line wavelengths. It was found that all flares with injected energy flux $>5$ F10 resulted in optically thick Si IV emission. Weaker flares also resulted in optical depth effects, depending on the other electron beam parameters. Empirically, optical depth effects occurred when the column mass was sufficiently enhanced, with a rough threshold column mass between $40<T<100 \mathrm{kK}$ of $m_{\mathrm{col}} \approx 5 \times 10^{-6} \mathrm{~g} \mathrm{~cm}^{-2}$.

The formation temperature in optically thin conditions was around $T \approx 60-100 \mathrm{kK}$, and in optically thick conditions it was somewhat lower, around $T \approx 30-60 \mathrm{kK}$. There were exceptions to this rule, where the Si IV resonance line cores formed in significantly higher temperatures $T>100 \mathrm{kK}$. When this occurred, the line cores were mostly optically thin and formed in a geometrically narrow region of significantly enhanced electron density (in excess of a few $\times 10^{13-14} \mathrm{~cm}^{-3}$ ).
Flares that deposit energy at higher altitudes than those in our simulations might also produce optically thick Si IV emission, even if the energy flux is relatively low. Longer flare loops might produce optically thick emission even with the lower range of injected flux, due to the larger column mass over which the Si IV populations could be enhanced.

A large-scale survey of SiIV emission during flares of various sizes, measuring intensities and line ratios and noting the existence or lack of any self-absorption features, should be undertaken. If observational evidence points toward optically thin emission, then we must address why modeling predicts the opposite. This is also relevant for the higher-than-observed intensities of other chromospheric synthetic spectral lines, such as $\mathrm{Mg}$ II $h$ and $k$ lines and C II resonance lines. As such, we also recommend adding to any observational effort the ratios of all the strong resonance lines observed by IRIS, which can be compared to simulations.

In these simulations the Si IV resonance lines show opacity effects during the heating phase and quickly revert to forming in optically thin conditions in the cooling phase. This will likely make detecting these effects on Si IV difficult in observations without adequate temporal cadence. Even IRIS flare observations with cadences of $t=5-10 \mathrm{~s}$ would only provide one or two profiles, from a particular spatial location during the heating phase, that exhibit the effects described here (assuming an energy deposition time of $t \sim 10 \mathrm{~s}$ as in our simulations). Subsequent observations from the same spatial location would be much weaker. Multithreaded-flare simulations (e.g., Reep et al. 2016) have shown that sustained heating of various threads into one atmospheric element can explain certain observations of Doppler motions. In such a scenario optical depth effects may be observational for longer as different threads are activated. The Si IV lines would also likely be weaker as only a fraction of threads would be activated at the same time, with emergent intensity from that pixel averaged over threads that are in their quiescent, heating, and cooling phases.

Finally, it is worth noting that the rapid cooling following cessation of flare energy input is likely to be, in part, due to an overestimation of thermal conduction. It was recently demonstrated (Bian et al. 2016a, 2016b, 2017, 2018; Emslie \& Bian 2018) that nonlocal effects and turbulence can lead to a thermal conduction that is significantly smaller than that predicted by classical Spitzer conductivity (even with saturation to the electron free-streaming limit as currently implemented in RADYN). We plan to implement the suppression of thermal conduction following Emslie \& Bian (2018) in a future work. This may extend the time period over which the Si IV resonance line exhibits optical depth effects.

Based on these simulations, we urge caution when analyzing observations of the SiIV resonance lines, as techniques that assume optically thin conditions may not be valid. Further, producing synthetic emission from simulated, or semi-empirical, flare atmospheres using a purely optically thin assumption, without NLTE radiation transfer, may not be appropriate. Modeling should include the effects of charge exchange, which is an important process in the formation of silicon ions in the solar atmosphere.

G.S.K. was funded by an appointment to the NASA Postdoctoral Program at Goddard Space Flight Center, 
administered by USRA through a contract with NASA. M.C. acknowledges support from the Research Council of Norway through its Centres of Excellence scheme, project no. 262622, and through grants of computing time from the Programme for Supercomputing. J.C.A. and A.N.D. acknowledge support from the IRIS mission. IRIS is a NASA small explorer mission developed and operated by LMSAL with mission operations executed at NASA Ames Research Center and major contributions to downlink communications funded by ESA and the Norwegian Space Centre. P.R.Y. acknowledges support from NASA grant NNX15AF25G. We appreciate the open data policies of the Opacity Project and the NIST and CHIANTI atomic database. The authors thank ISSI and ISSIBJ for the support to the teams "Diagnosing Heating Mechanisms in Solar Flares through Spectroscopic Observations of Flare Ribbons" and "New Diagnostics of Particle Acceleration in Solar Coronal Nanoflares from Chromospheric Observations and Modeling." Finally, we would like to thank the anonymous referee, whose comments helped to improve this work.

\section{Appendix A \\ Silicon Model Atom Details}

Here we present details regarding the construction of our model silicon atom.

Oscillator strengths, $f_{i j}$, were taken from the CHIANTI database. Radiative damping coefficients were obtained by summing the Einstein $A_{j i}$-coefficients associated with upper level $j$, so that the lifetime of level $j$ was $t_{j}=1 / \sum A_{j i}$. Direct collisional ionization rate coefficients and collisional excitation rate coefficients were taken from CHIANTI.

The abundance used was $A_{\mathrm{Si}}=7.51$ (Asplund et al. 2009), defined on the usual logarithmic scale, with $A_{\mathrm{H}}=12$. This is assumed to be constant through the loop and in time. We note that recent work by Olluri et al. (2015) and Martínez-Sykora et al. (2016) argues in favor of using coronal abundances for silicon, based on the findings that first ionization potential (FIP) effects enrich the abundance of low-FIP elements in the upper atmosphere (e.g., Feldman 1992; Laming 2004). However, Warren et al. (2016a) recently reported that in impulsive heating events low-FIP elements have a composition similar to the photosphere. Since we are simulating flares, and given general ambiguity in elemental abundances, we elect to use the photospheric value (Asplund et al. 2009), but we provide in Appendix B an illustration of the impact on our results of using the coronal abundance.

Photoionization cross sections from the TOPbase database ${ }^{6}$ (Cunto et al. 1993), remapped to the wavelengths in the RADYN background opacity package, were used for the 33 bound-free transitions. To convert the TOPbase transitions from energy in rydberg units to wavelengths in angstroms, we used the value of the Rydberg constant for silicon, $R_{\mathrm{Si}}=109735.1723 \mathrm{~cm}^{-1}$ $\left(R_{\mathrm{Si}}=R_{\infty} /\left(1+m_{e} / M_{\mathrm{Si}}\right)\right.$, for electron mass $m_{e}$, atomic mass $M_{\mathrm{Si}}$, and Rydberg constant for infinite mass $R_{\infty}$ ) and corrected the threshold energies according to the NIST database (Kramida et al. 2018), in the expression

$$
\lambda[\AA]=\frac{1 \times 10^{8}}{R_{\mathrm{Si}}\left(\left.E[\mathrm{Ryd}]\right|_{\mathrm{TOPbase}}+\Delta E[\mathrm{Ryd}]\right)},
$$

\footnotetext{
6 http://cdsweb.u-strasbg.fr/topbase/topbase.html
}

where $\Delta E$ is the difference in NIST threshold energy and the TOPbase value. As noted in Leenaarts et al. (2013), TOPbase provides photoionization cross sections that extend below the threshold energy, which we discard here.

Recipes from Arnaud \& Rothenflug (1985) were used for charge exchange (also known as charge transfer) with H I, H II, $\mathrm{He}$ I, and He II.

For the 33 detailed bound-free transitions the TOPbase cross sections were used for radiative recombinations (the inverse of photoionizations). Only considering radiative recombinations to the levels treated in detail can risk underestimating the total recombination rate. To account for these additional radiative recombinations, and to include dielectronic recombinations, we used rate coefficients from the CHIANTI database in the following way. First, we computed the total radiative recombination rate coefficient to each charge state as a function of temperature at $t=0 \mathrm{~s}$ in an MS_RADYN test simulation. Then, we computed the difference between the total recombination rate coefficients to each charge state from CHIANTI (radiative plus dielectronic) and the radiative recombinations treated in detail by MS_RADYN. This excess was then included as an additional recombination term in our model atom for flare simulations, taking place to the ground terms and excited states of the relevant charge state, according to their statistical weights.

Note that we do not include suppression of dielectronic recombination, a process that occurs at high densities (e.g., Summers 1974; Nikolić et al. 2013; Young 2018; Young et al. 2018a, and references therein). It has been shown that densitysensitive dielectronic recombination can lead to a higher Si IV fraction at lower temperatures and a higher peak fraction, compared to the zero-density limit assumed by the CHIANTI rates (e.g., Polito et al. 2016). RADYN has an implementation of density effects according to Summers (1974; see, e.g., Rathore $\&$ Carlsson 2015), but future iterations of this work will modify RADYN to also accept dielectronic rate coefficients as functions of density, with density-dependent rate coefficients from the $\mathrm{ADAS}^{7}$ atomic database included in place of those from CHIANTI. For now, we focus on the effects of photoionizations, charge exchange, and opacity but include an illustration of how suppression of dielectronic recombination might affect our results in Appendix $\mathrm{C}$, by performing an experiment using the suppression factors of Nikolić et al. (2013) as implemented by Young (2018).

The silicon data that we used from CHIANTI were from CHIANTI V8.0.7. The sources of those data were Dere (2007), Tayal (2008), Abdel-Naby et al. (2012), Dufton \& Kingston (1989), Altun et al. (2006, 2007), Liang et al. (2009), Badnell (2006), and Zatsarinny et al. (2004).

\section{Appendix B Using Coronal Abundances}

In some recent work (e.g., Olluri et al. 2015; MartínezSykora et al. 2016) it has been argued that it is appropriate to use an abundance larger than the photospheric value for silicon and other low-FIP elements (Laming 2004), for example, the coronal abundance from Feldman (1992). Warren et al. (2016a) demonstrate that it is appropriate to use photospheric abundances for impulsive heating events, so we contend that our use of the Asplund et al. (2009) photospheric abundance is

\footnotetext{
http://open.adas.ac.uk
} 

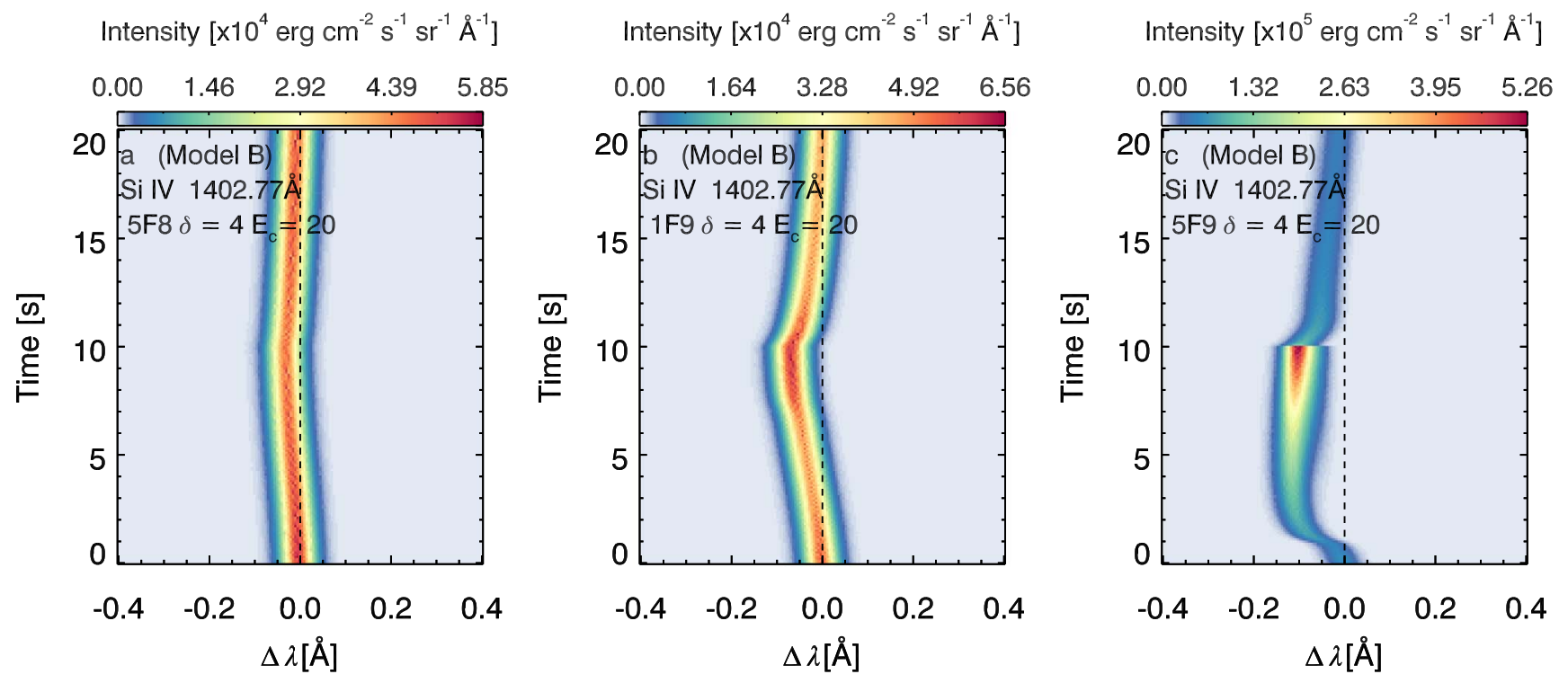

Intensity $\left[\times 10^{6} \mathrm{erg} \mathrm{cm}^{-2} \mathrm{~s}^{-1} \mathrm{sr}^{-1} \AA^{-1}\right]$

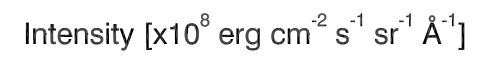

Intensity $\left[\times 10^{8} \mathrm{erg} \mathrm{cm}^{-2} \mathrm{~s}^{-1} \mathrm{sr}^{-1} \AA^{-1}\right]$
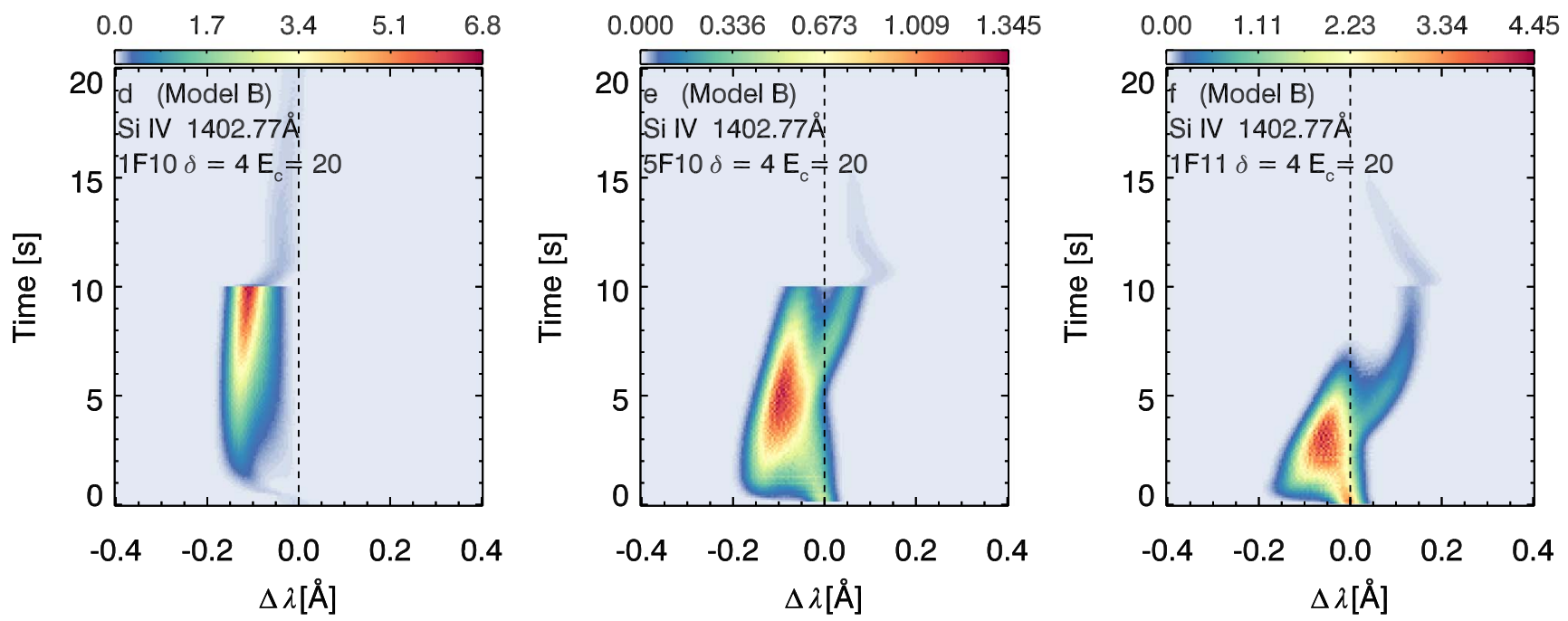

Figure 12. Comparing the evolution of the Model B line profiles shown in Figure 4 and instead using the coronal abundance $A_{\mathrm{Si} \text {,Feld }}=8.10$. Panels and simulations are as described in Figure 4.

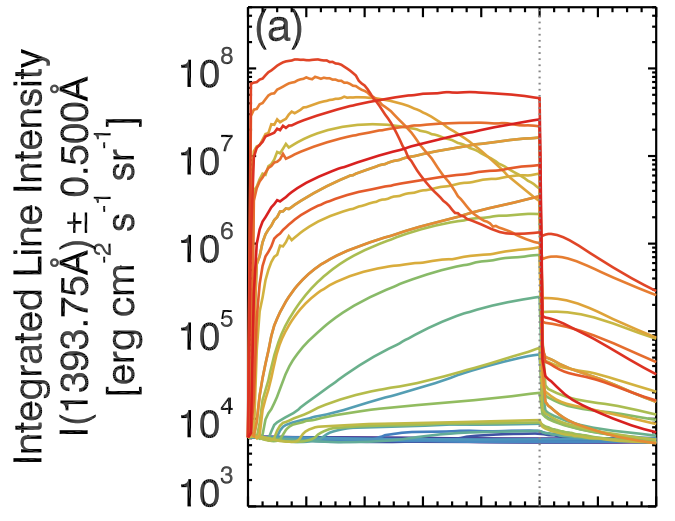

Model B

$\begin{array}{llllllll}0 & 2 & 4 & 6 & 8 & 10 & 12 & 14\end{array}$

Feldman 1992 Abund.
Time [s]

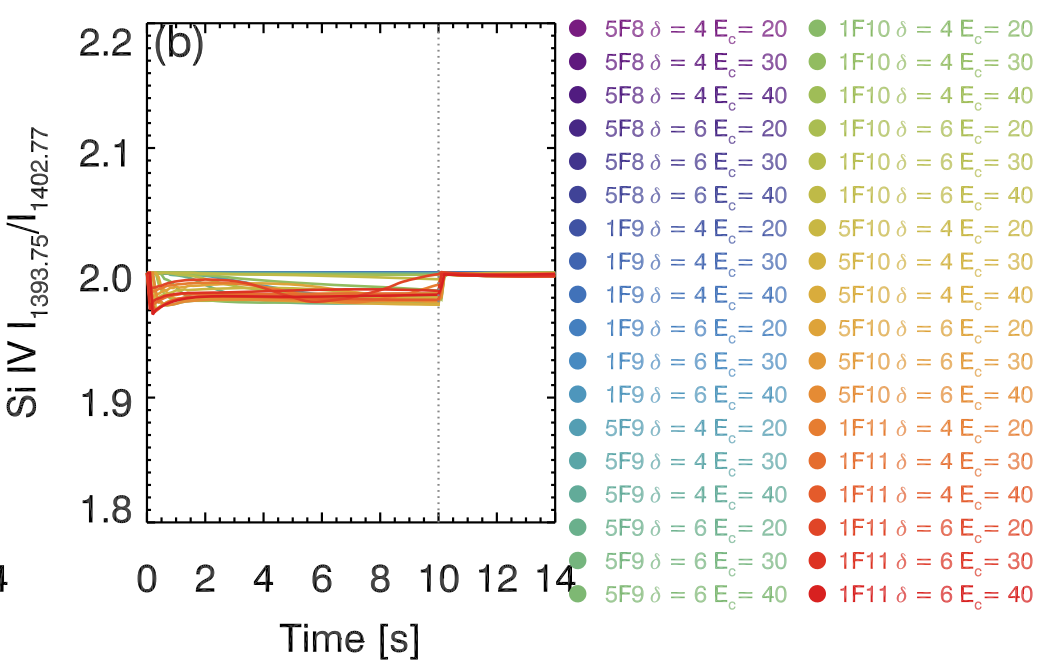

Figure 13. Same as Figure 6, but showing results as computed using the optically thin assumption via Model B with coronal abundance $A_{\mathrm{Si} \text {,Feld }}=8.10$. 
valid, particularly given the general ambiguity of elemental abundances in flares. Nevertheless, it is useful to provide an illustration of the impact on our results of instead using coronal abundances. Model $\mathrm{B}$ results were all recomputed using silicon coronal abundances from Feldman (1992), $A_{\mathrm{Si} \text {,Feld }}=$ 8.10. Since MS_RADYN is more computationally demanding, we instead ran some test cases using the coronal abundance: $5 \mathrm{~F} 10 \delta 6 E_{\mathrm{c}} 20$ and $1 \mathrm{~F} 10 \delta 4 E_{\mathrm{c}} 30$.

Figure 12 shows the same Model B simulations presented in Figure 4 , but using $A_{\mathrm{Si} \text {,Feld }}$. The line shapes, Doppler motions, and general behavior are preserved, but the lines are more intense. This can also be seen by comparing light curves using $A_{\mathrm{Si} \text {,Feld }}$ (Figure 13) and the photospheric value (Figure 7). Again, while temporal behavior is preserved, the coronal abundances result in much more intense emission. The line ratios are unchanged.

Results from MS_RADYN were similarly affected, with the line intensity becoming larger when using the coronal abundance values. The larger abundance also results in the $t=0 \mathrm{~s}$ atmosphere being somewhat more optically thick (so that the line forms closer to $\tau_{\nu} \approx 0.1$ ) and results in the $1 \mathrm{~F} 10 \delta 4 E_{\mathrm{c}} 30$ simulation exhibiting optical depth effects in a narrow region of the line core.

\section{Appendix C \\ Suppression of Dielectronic Recombination}

At high densities, dielectronic recombination rates can be suppressed over the zero-density limits typically used (e.g., the rates in CHIANTI), due to collisions depopulating the higherlying levels through which dielectronic recombination occurs. This is discussed in detail by, for example, Summers (1974), Nikolić et al. (2013), Judge (2015), and Young (2018).

While some atomic databases such as ADAS do contain density-sensitive dielectronic recombination rate coefficients, implementing these in RADYN or MS_RADYN requires modifications to the ways in which the codes deal with atomic data. This will be the focus of future research, but here we demonstrate the potential impact of suppression of dielectronic recombination on our results.

We use an approximation similar to that of Young (2018), who used suppression factors based on the work of Nikolić et al. (2013). The isoelectronic sequence, charge, electron density, and temperature are used in the expressions of Nikolić et al. (2013) to derive a suppression factor, which we used to suppress the dielectronic rates from CHIANTI in our model atom Si30_atom_wchex. This is somewhat artificial in our flare atmospheres, as the density is assumed constant for all locations and times when computing the suppressed rates, but is suitable for illustrative purposes. An electron density of $n_{e}=1 \times 10^{12} \mathrm{~cm}^{-3}$ was chosen. While this is too large for the quiet Sun and the electron density in the flaring TR varies with flare strength, the results of Polito et al. (2016) show

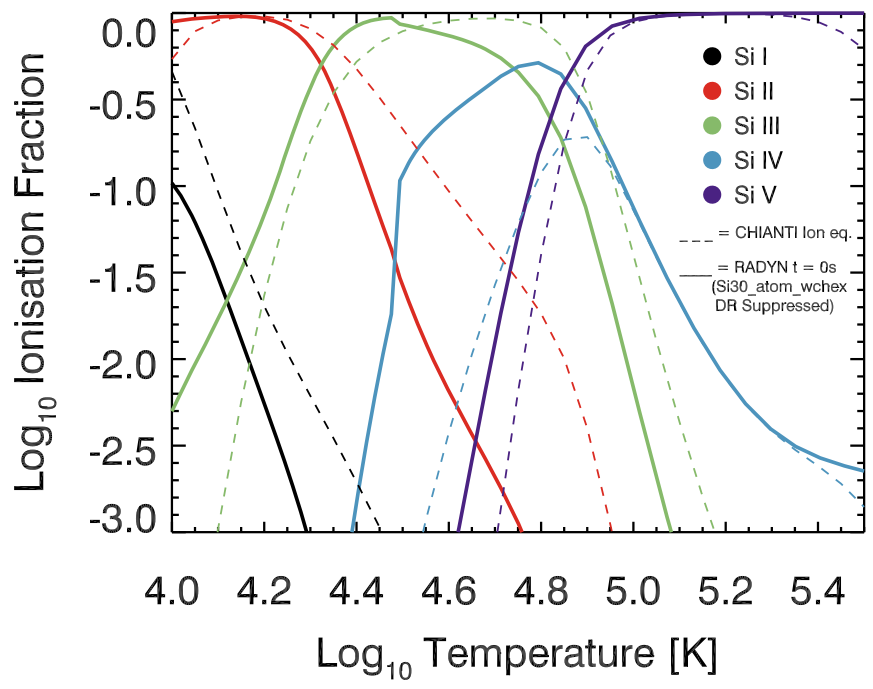

Figure 14. Same as Figure 1, but showing the effects of including suppression of dielectronic recombination with the Si30_atom_wchex atom.

that the differences between $n_{e}=\left[1 \times 10^{11}, 1 \times 10^{12}, 1 \times\right.$ $\left.10^{13}\right] \mathrm{cm}^{-3}$ are smaller than the jump from the zero-density limit that was used in the rest of this study. A mid-range value of $n_{e}=1 \times 10^{12} \mathrm{~cm}^{-3}$ therefore seems like an appropriate choice.

Two MS_RADYN test cases were run with the suppressed dielectronic rates: one moderate flare $\left(5 \mathrm{~F} 10 \delta 6 E_{\mathrm{c}} 20\right)$, and one weaker flare $\left(1 \mathrm{~F} 10 \delta 4 E_{\mathrm{c}} 30\right)$. The latter simulation was chosen to also determine whether using the suppression of dielectronic recombination would result in opacity effects becoming important (they were not when using the zero-density limit). The ionization fractions at $t=0 \mathrm{~s}$ are shown in Figure 14, indicating that with suppressed rates Si IV forms over a similar temperature range as the zero-density limit, but with a peak temperature that has shifted lower to $\log T=4.77$ $(T=59.3 \mathrm{kK})$ and optical depth $\tau_{\lambda} \approx 0.03$. The peak of the $\mathrm{Si} I V$ fraction also increased in magnitude (compare to Figure 1(a)). This results in an increased intensity of emission during the flares, but the line shapes and features are largely unchanged. The most intense emission is $\approx 1.3 \times$ larger in the $1 \mathrm{~F} 10 \delta 4 E_{\mathrm{c}} 30$ simulation and $\approx 2.9 \times$ larger in the $5 \mathrm{~F} 10 \delta 6 E_{\mathrm{c}} 20$ simulation. Figures 15(a)-(d) show wavelength versus time images for the cases without suppression of dielectronic recombination (panels (a), (b)) and with suppression (panels (c), (d)). Overall the optical depth effects on the line formation were unchanged.

Future efforts will attempt to include suppression of dielectronic recombination self-consistently in RADYN and MS_RADYN, using the actual atmospheric density with rate coefficients tabulated as functions of both temperature (as currently implemented) and electron density. 

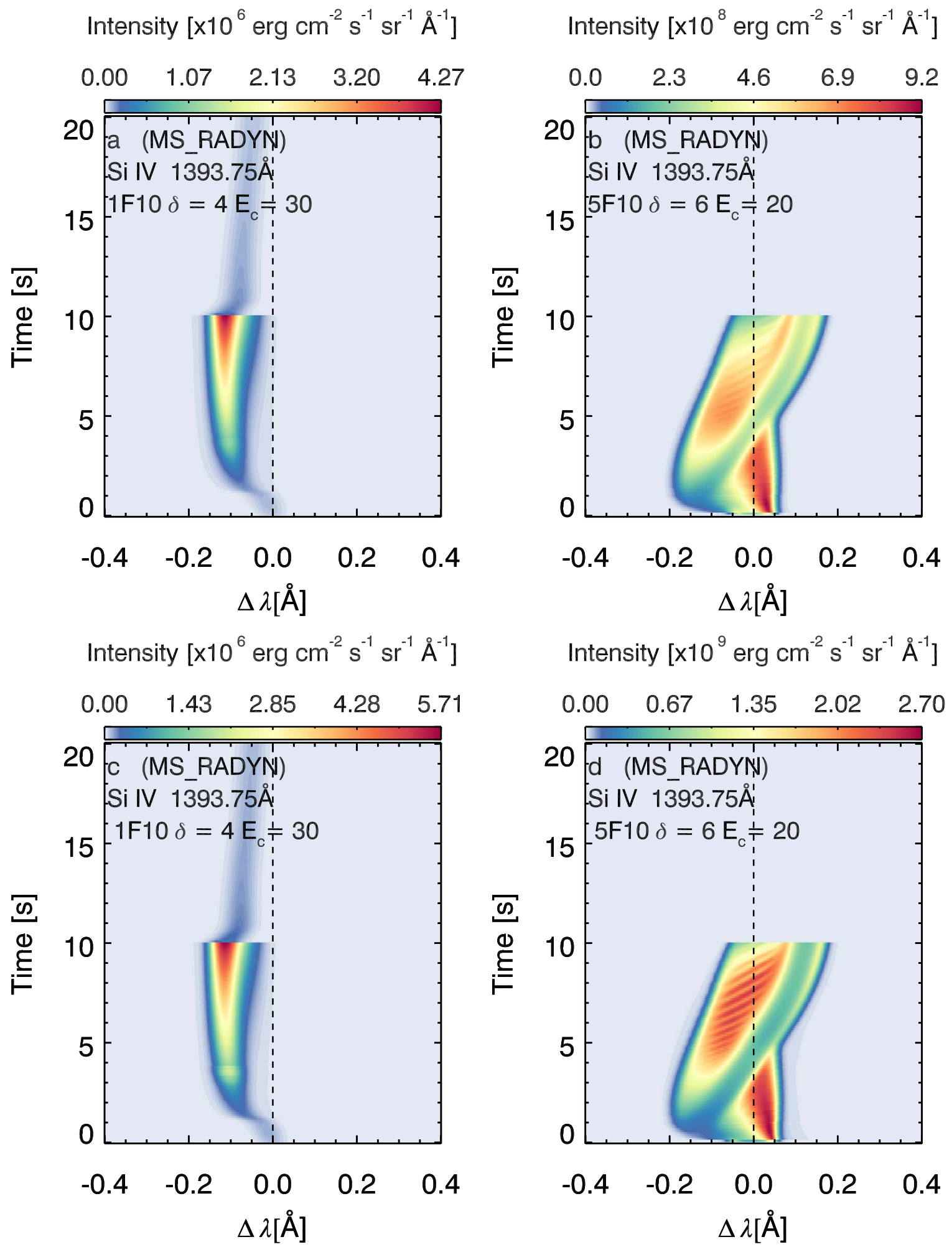

Figure 15. Comparing Si IV $\lambda 1393$ line emission without suppression of dielectronic recombination (panels (a), (b)) and with suppression (panels (c), (d)) for two test cases of MS_RADYN using Si30_atom_wchex. Panels are as described in Figure 4.

\section{ORCID iDs}

Graham S. Kerr (iD https://orcid.org/0000-0001-5316-914X Mats Carlsson (ib https://orcid.org/0000-0001-9218-3139 Joel C. Allred (ib https://orcid.org/0000-0003-4227-6809 Peter R. Young (iD https://orcid.org/0000-0001-9034-2925

\section{References}

Abbett, W. P., \& Hawley, S. L. 1999, ApJ, 521, 906

Abdel-Naby, S. A., Nikolić, D., Gorczyca, T. W., Korista, K. T., \& Badnell, N. R. 2012, A\&A, 537, A40

Allred, J. C., Hawley, S. L., Abbett, W. P., \& Carlsson, M. 2005, ApJ, 630,573 
Allred, J. C., Kowalski, A. F., \& Carlsson, M. 2015, ApJ, 809, 104

Altun, Z., Yumak, A., Badnell, N. R., Loch, S. D., \& Pindzola, M. S. 2006 A\&A, 447, 1165

Altun, Z., Yumak, A., Yavuz, I., et al. 2007, A\&A, 474, 1051

Arnaud, M., \& Rothenflug, R. 1985, A\&AS, 60, 425

Asplund, M., Grevesse, N., Sauval, A. J., \& Scott, P. 2009, ARA\&A, 47, 481

Badnell, N. R. 2006, ApJS, 167, 334

Bartoe, J.-D. F., \& Brueckner, G. E. 1975, JOSA, 65, 13

Bian, N., Emslie, A. G., Horne, D., \& Kontar, E. P. 2018, ApJ, 852, 127

Bian, N. H., Emslie, A. G., \& Kontar, E. P. 2017, ApJ, 835, 262

Bian, N. H., Kontar, E. P., \& Emslie, A. G. 2016a, ApJ, 824, 78

Bian, N. H., Watters, J. M., Kontar, E. P., \& Emslie, A. G. 2016b, ApJ, 833, 76

Bloomfield, D. S., Mathioudakis, M., Christian, D. J., Keenan, F. P., \& Linsky, J. L. 2002, A\&A, 390, 219

Brannon, S. R., Longcope, D. W., \& Qiu, J. 2015, ApJ, 810, 4

Brekke, P., Kjeldseth-Moe, O., Bartoe, J.-D. F., \& Brueckner, G. E. 1991, ApJS, 75, 1337

Brown, J. C. 1971, SoPh, 18, 489

Brown, S. A., Fletcher, L., Kerr, G. S., et al. 2018, arXiv:1807.03373

Carlsson, M. 1998, in Space Solar Physics: Theoretical and Observational Issues in the Context of the SOHO Mission, ed. J. C. Vial, K. Bocchialini, \& P. Boumier (Berlin: Springer), 163

Carlsson, M., \& Stein, R. F. 1992, ApJL, 397, L59

Carlsson, M., \& Stein, R. F. 1997, ApJL, 481, 500

Christian, D. J., Mathioudakis, M., Bloomfield, D. S., et al. 2006, A\&A, 454, 889

Cunto, W., Mendoza, C., Ochsenbein, F., \& Zeippen, C. J. 1993, A\&A, $275, \mathrm{~L} 5$

Dame, L., \& Vial, J.-C. 1985, ApJL, 299, L103

De Pontieu, B., Title, A. M., Lemen, J. R., et al. 2014, SoPh, 289, 2733

Dere, K. P. 2007, A\&A, 466, 771

Dere, K. P., Landi, E., Mason, H. E., Monsignori Fossi, B. C., \& Young, P. R. 1997, A\&AS, 125, 149

Dorfi, E. A., \& Drury, L. O. 1987, JCoPh, 69, 175

Doschek, G. A., Feldman, U., Kreplin, R. W., \& Cohen, L. 1980, ApJ, 239,725

Doschek, G. A., Warren, H. P., \& Young, P. R. 2016, ApJ, 832, 77

Dudík, J., Del Zanna, G., Dzifčáková, E., Mason, H. E., \& Golub, L. 2014, ApJL, 780, L12

Dudík, J., Polito, V., Dzifčáková, E., Del Zanna, G., \& Testa, P. 2017, ApJ, 842,19

Dufton, P. L., \& Kingston, A. E. 1989, MNRAS, 241, 209

Dzifčáková, E., \& Dudík, J. 2018, A\&A, 610, A67

Dzifčáková, E., Dudík, J., \& Mackovjak, Š 2016, A\&A, 589, A68

Dzifčáková, E., Vocks, C., \& Dudík, J. 2017, A\&A, 603, A14

Emslie, A. G. 1978, ApJ, 224, 241

Emslie, A. G., \& Bian, N. H. 2018, ApJ, 865, 1

Fang, C., Henoux, J. C., \& Gan, W. Q. 1993, A\&A, 274, 917

Feldman, U. 1992, PhyS, 46, 202

Fisher, G. H. 1989, ApJ, 346, 1019

Fisher, G. H., Canfield, R. C., \& McClymont, A. N. 1985a, ApJ, 289, 414

Fisher, G. H., Canfield, R. C., \& McClymont, A. N. 1985b, ApJ, 289, 425

Fisher, G. H., Canfield, R. C., \& McClymont, A. N. 1985c, ApJ, 289, 434

Fletcher, L., Dennis, B. R., Hudson, H. S., et al. 2011, SSRv, 159, 19

Fletcher, L., \& Hudson, H. S. 2008, ApJ, 675, 1645

Graham, D. R., \& Cauzzi, G. 2015, ApJL, 807, L22

Gustafsson, B. 1973, UppAn, 5, 6

Holman, G. D., Aschwanden, M. J., Aurass, H., et al. 2011, SSRv, 159, 107

Hoyng, P., van Beek, H. F., \& Brown, J. C. 1976, SoPh, 48, 197

Jaeggli, S. A., Judge, P. G., \& Daw, A. N. 2018, ApJ, 855, 134

Judge, P. G. 2015, ApJ, 808, 116
Judge, P. G., Carlsson, M., \& Stein, R. F. 2003, ApJ, 597, 1158

Kerr, G. S. 2017, PhD thesis, Univ. Glasgow

Kerr, G. S., Fletcher, L., Russell, A. J. B., \& Allred, J. C. 2016, ApJ, 827, 101

Kerr, G. S., Simões, P. J. A., Qiu, J., \& Fletcher, L. 2015, A\&A, 582, A50

Kramida, A., Ralchenko, Y., Reader, J. \& NIST ASD Team 2018, NIST Atomic Spectra Database, (ver. 5.5.6), (Online) Available: https://physics. nist.gov/asd (2018 May 14) National Institute of Standards and Technology, Gaithersburg, MD

Kuridze, D., Mathioudakis, M., Simões, P. J. A., et al. 2015, ApJ, 813, 125

Laming, J. M. 2004, ApJ, 614, 1063

Landi, E., Young, P. R., Dere, K. P., Del Zanna, G., \& Mason, H. E. 2013, ApJ, 763, 86

Leenaarts, J., Pereira, T. M. D., Carlsson, M., Uitenbroek, H., \& De Pontieu, B. 2013, ApJ, 772, 89

Li, Y., Kelly, M., Ding, M. D., et al. 2017, ApJ, 848, 118

Liang, G. Y., Whiteford, A. D., \& Badnell, N. R. 2009, A\&A, 500, 1263

Lin, H.-H., \& Carlsson, M. 2015, ApJ, 813, 34

Lin, R. P., Dennis, B. R., Hurford, G. J., et al. 2002, SoPh, 210, 3

Magain, P. 1986, A\&A, 163, 135

Martínez-Sykora, J., De Pontieu, B., Hansteen, V. H., \& Gudiksen, B. 2016, ApJ, 817, 46

Mathioudakis, M., McKenny, J., Keenan, F. P., Williams, D. R., \& Phillips, K. J. H. 1999, A\&A, 351, L23

Meegan, C., Lichti, G., Bhat, P. N., et al. 2009, ApJ, 702, 791

Milligan, R. O., \& Dennis, B. R. 2009, ApJ, 699, 968

Milligan, R. O., Gallagher, P. T., Mathioudakis, M., et al. 2006, ApJL, 638, L117

Nelson, C. J., Freij, N., Reid, A., et al. 2017, ApJ, 845, 16

Nikolić, D., Gorczyca, T. W., Korista, K. T., Ferland, G. J., \& Badnell, N. R. 2013, ApJ, 768, 82

Nóbrega-Siverio, D., Martínez-Sykora, J., Moreno-Insertis, F., \& Rouppe van der Voort, L. 2017, ApJ, 850, 153

Olluri, K., Gudiksen, B. V., Hansteen, V. H., \& De Pontieu, B. 2015, ApJ, 802,5

Peter, H., Tian, H., Curdt, W., et al. 2014, Sci, 346, 1255726

Polito, V., Del Zanna, G., Dudík, J., et al. 2016, A\&A, 594, A64

Polito, V., Testa, P., Allred, J., et al. 2018, ApJ, 856, 178

Rathore, B., \& Carlsson, M. 2015, ApJ, 811, 80

Reep, J. W., Polito, V., Warren, H. P., \& Crump, N. A. 2018a, ApJ, 856, 149

Reep, J. W., \& Russell, A. J. B. 2016, ApJL, 818, L20

Reep, J. W., Russell, A. J. B., Tarr, L. A., \& Leake, J. E. 2018b, ApJ, 853, 101

Reep, J. W., Warren, H. P., Crump, N. A., \& Simões, P. J. A. 2016, ApJ, 827,145

Rubio da Costa, F., \& Kleint, L. 2017, ApJ, 842, 82

Smith, D. F., \& Auer, L. H. 1980, ApJ, 238, 1126

Summers, H. P. 1974, Appleton Laboratory Internal Memorandum IM367 \& Re-Issued with Improvements as AL-R-5., Tech. Rep., IM367

Tayal, S. S. 2008, ApJS, 179, 534

Tian, H., Young, P. R., Reeves, K. K., et al. 2015, ApJ, 811, 139

Uitenbroek, H. 2001, ApJ, 557, 389

Uitenbroek, H. 2002, ApJ, 565, 1312

Warren, H. P., Brooks, D. H., Doschek, G. A., \& Feldman, U. 2016a, ApJ, 824,56

Warren, H. P., Reep, J. W., Crump, N. A., \& Simões, P. J. A. 2016b, ApJ, 829,35

Yan, L., Peter, H., He, J., et al. 2015, ApJ, 811, 48

Young, P. R. 2018, ApJ, 855, 15

Young, P. R., Keenan, F. P., Milligan, R. O., \& Peter, H. 2018a, ApJ, 857, 5

Young, P. R., Tian, H., Peter, H., et al. 2018b, arXiv: 1805.05850

Zatsarinny, O., Gorczyca, T. W., Korista, K., Badnell, N. R., \& Savin, D. W. 2004, A\&A, 426, 699 